\title{
An environmentally safe condition for the synthesis of aryl and alkyl sulfonyl hydrazones via one-pot reaction $\uparrow$
}

Micael Rodrigues Cunha ${ }^{\S a}$, Maurício Temotheo Tavares ${ }^{\S a}$, Camila Félix Carvalho ${ }^{a}$, Nuno Albuquerque T. Silva ${ }^{a}$, Alfredo Danilo F. Souza ${ }^{a}$, Gustavo José V. Pereira ${ }^{a}$, Fábio Furlan Ferreira $^{\mathrm{b}}$, Roberto Parise-Filho*a

a. Laboratory of Design and Synthesis of Bioactive Substances (LAPESSB), Faculty of Pharmaceutical Sciences, University of São Paulo, São Paulo, SP, Brazil

b. Center of Natural and Human Sciences (CCNH), Federal University of ABC (UFABC), Santo André, SP, Brazil

$\S$ Micael Rodrigues Cunha and Maurício Temotheo Tavares contributed equally to this work

*E-mail: roberto.parise@usp.br (R. Parise-Filho); +55 113091.3793

\section{$\dagger$ †UPPORTING INFORMATION}

\author{
Table of contents
}

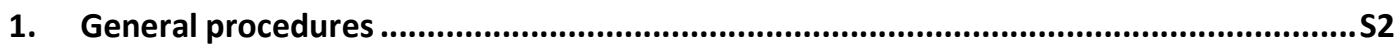

1.1 Synthesis of aryl/alkylsulfonyl hydrazones derivatives....................................... S2

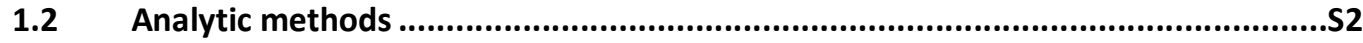

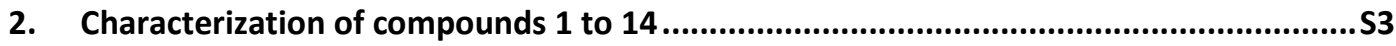

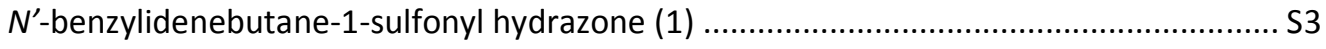

$N^{\prime}$-(4-methoxybenzylidene) butane-1- sulfonyl hydrazone (2)....................................... S3

$N^{\prime}$-(4-nitrobenzylidene) butane-1- sulfonyl hydrazone (3) .............................................. S3

$N^{\prime}$-(4-hydroxy-3-methoxybenzylidene) butane-1- sulfonyl hydrazone (4) ........................ S3

$N^{\prime}$-(thiophen-2-ylmethylene) butane-1- sulfonyl hydrazone (5)..................................... S3

$N^{\prime}$-benzylidene-ethane-sulfonyl hydrazone (6).............................................................. S4

$N^{\prime}$-(4-methoxybenzylidene) ethane-sulfonyl hydrazone (7) ........................................... S4

$N^{\prime}$-(4-nitrobenzylidene) ethane-sulfonyl hydrazone (8) ................................................. S4

$N^{\prime}$-(4-hydroxy-3-methoxybenzylidene) ethanesulfonyl hydrazone (9) .............................. S4

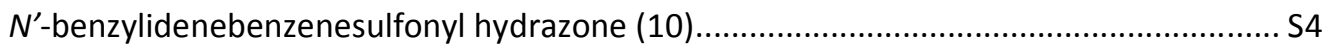


$N^{\prime}$-(4-methoxybenzylidene) benzenesulfonyl hydrazone (11)

$N^{\prime}$-(4-nitrobenzylidene) benzenesulfonyl hydrazone (12) ...................................... S4

$N^{\prime}$-(4-hydroxy-3-methoxybenzylidene) benzenesulfonyl hydrazone (13) ..................... S5

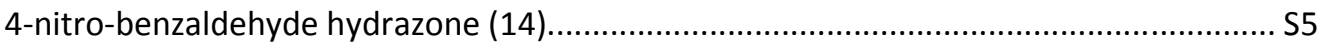

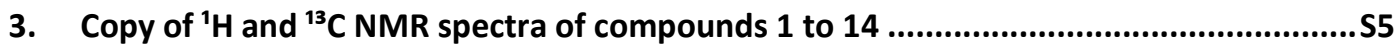

4. X-ray powder diffraction measurements .............................................................S199

4.1 Structure determination procedure ......................................................... S199

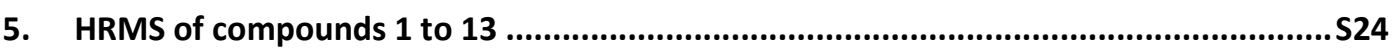

$N^{\prime}$-benzylidenebutane-1-sulfonyl hydrazone (1) ................................................... S34

$N^{\prime}$-(4-methoxybenzylidene) butane-1- sulfonyl hydrazone (2) .................................. S34

$N^{\prime}$-(4-nitrobenzylidene) butane-1- sulfonyl hydrazone (3) ....................................... S25

$N^{\prime}$-(4-hydroxy-3-methoxybenzylidene) butane-1- sulfonyl hydrazone (4) ................... S26

$N^{\prime}$-(thiophen-2-ylmethylene) butane-1- sulfonyl hydrazone (5).............................. S26

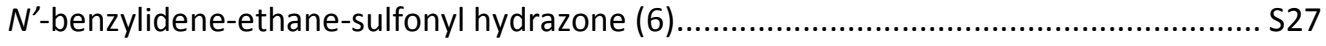

$N^{\prime}$-(4-methoxybenzylidene) ethane-sulfonyl hydrazone (7) ..................................S28

$N^{\prime}$-(4-nitrobenzylidene) ethane-sulfonyl hydrazone (8) ...................................... S28

$N^{\prime}$-(4-hydroxy-3-methoxybenzylidene) ethanesulfonyl hydrazone (9) ........................ S29

$N^{\prime}$-benzylidenebenzenesulfonyl hydrazone (10)................................................. S30

$N^{\prime}$-(4-methoxybenzylidene) benzenesulfonyl hydrazone (11) .................................. S30

$N^{\prime}$-(4-nitrobenzylidene) benzenesulfonyl hydrazone (12) ......................................... S31

$N^{\prime}$-(4-hydroxy-3-methoxybenzylidene) benzenesulfonyl hydrazone (13) .................... S32

\section{General procedures}

\subsection{Synthesis of aryl/alkylsulfonyl hydrazones derivatives}

A solution of hydrazine hydrate $(0.6 \mathrm{mmol}, 1.2$ equiv) in ethanol $(1 \mathrm{~mL})$ was dropwise added to a cold solution of the sulfonyl chloride $(0.5 \mathrm{mmol})$ in ethanol $(1 \mathrm{~mL})$, at $0{ }^{\circ} \mathrm{C}$. Thus, triethylamine $(1.0 \mathrm{mmol}, 2$ equiv.) was dropwise added. The mixture stirred at room temperature for $1 \mathrm{~h}$. The reaction was monitored by thin-layer chromatography (TLC) using hexane/ethyl acetate (1:1) as eluent. After complete consumption of sulfonyl chloride, a solution of aldehyde ( $0.72 \mathrm{mmol}, 1.2$ equiv.) in ethanol $(1 \mathrm{~mL})$ was dropwise added followed by acidification of the medium until $\mathrm{pH} 4.0$ with some drops of $\mathrm{HCl}$. Indicator strips monitored the $\mathrm{pH}$ of the medium. After acidification, the stirring solution was kept at $60^{\circ} \mathrm{C}$ for 6 to 9 hours to afford the desired sulfonyl hydrazone.

\subsection{Analytic methods}

${ }^{1} \mathrm{H}$ and ${ }^{13} \mathrm{C}$ Nuclear Magnetic Resonance (NMR). The ${ }^{1} \mathrm{H}$ and ${ }^{13} \mathrm{C}$ NMR spectra were recorded using a Bruker Advanced DPX-300 spectrometer at $300 \mathrm{MHz}$ and $75 \mathrm{MHz}$, respectively. Chemical shifts were expressed as $\delta$ units, using tetramethylsilane (TMS) as internal standard, and $\mathrm{CDCl}_{3}, \mathrm{DMSO}-d 6$ or Acetone- $d 6$ as solvents. The spectral splitting 
patterns are described as follows: $s$, singlet; $d$, doublet; dd, double doublet; $q$, quartet; $t$, triplet; $m$, multiplet peak.

Melting Point (mp). The melting points were determined in open capillary tubes using a Büchi M-565 digital melting point apparatus and were uncorrected.

High Resolution Mass Spectrometry (HRMS). HRMS were measured with a MicroToF Bruker Daltonics spectrometer with an ESI source coupled to a Waters HPLC system.

X-ray Powder Diffraction (XRPD). Suitable crystals of $N^{\prime}$-(4-methoxybenzylidene) benzenesulfonyl hydrazone were obtained from ethyl acetate solution for structural characterization. Crystal structure determination was carried out on a STADI-P diffractometer (Stoe ${ }^{\circledR}$, Darmstadt, Germany) operating in transmission geometry using a $\mathrm{K}_{1}$ radiation $(\lambda=$ $0.7093 \AA$ ) , wavelength emitted by a Mo anode and selected by a curved Ge (111) crystal, with a tube voltage of $50 \mathrm{kV}$ and a current of $40 \mathrm{~mA}$. The sample was packed between two acetatecellulose foils, and the sample holder was held spinning during data collection. The diffracted intensities were collected by a silicon microstrip detector, Mythen $1 \mathrm{~K}$ (Dectris ${ }^{\circledR}$, Baden, Switzerland), in the range from $1.6^{\circ}$ to $50.7^{\circ}$, with $200 \mathrm{~s}$ of integration time at each $0.785^{\circ}$.

\section{Characterization of compounds 1 to 14}

N'-benzylidenebutane-1-sulfonyl hydrazone (1)

Yellow resin, 90\% yield $(0.216 \mathrm{~g}) .{ }^{1} \mathrm{H}$ NMR $\left(300 \mathrm{MHz}^{\mathrm{CDCl}} \mathrm{CD}_{3}\right) \delta: 8.35(\mathrm{~s}, 1 \mathrm{H}), 7.86(\mathrm{~s}, 1 \mathrm{H})$, $7.65\left(\mathrm{dd}, J^{1}=6.5, J^{2}=2.9 \mathrm{~Hz}, 2 \mathrm{H}\right), 7.38(\mathrm{t}, 3 \mathrm{H}), 3.31(\mathrm{t}, 2 \mathrm{H}), 1.85(\mathrm{~m}, 2 \mathrm{H}), 1.47(\mathrm{~m}, 2 \mathrm{H})$, 0.94 (t, $3 \mathrm{H}) .{ }^{13} \mathrm{C} \mathrm{NMR}\left(75 \mathrm{MHz}, \mathrm{CDCl}_{3}\right) \delta: 147.7,133.3,130.6,128.8,127.5,55.1,25.3,21.6$, 13.6. HRMS (ESI) $\mathrm{m} / z$ calcd for $\mathrm{C}_{11} \mathrm{H}_{16} \mathrm{~N}_{2} \mathrm{O}_{2} \mathrm{~S}\left(\mathrm{M}^{+}\right)$240.0932, found $263.0822\left([\mathrm{M}+\mathrm{Na}]^{+}\right)$; $241.0993\left([\mathrm{M}+\mathrm{H}]^{+}\right)$.

\section{N'-(4-methoxybenzylidene) butane-1- sulfonyl hydrazone (2)}

Yellow resin, 91\% yield (0.246 g). ${ }^{1} \mathrm{H}$ NMR $\left(300 \mathrm{MHz}, \mathrm{CDCl}_{3}\right) \delta: 8.12(\mathrm{~s}, 1 \mathrm{H}), 7.81(\mathrm{~s}, 1 \mathrm{H})$, $7.59(\mathrm{~d}, J=8.7 \mathrm{~Hz}, 2 \mathrm{H}), 6.89(\mathrm{~d}, J=8.7 \mathrm{~Hz}, 2 \mathrm{H}), 3.83(\mathrm{~s}, 3 \mathrm{H}), 3.29(\mathrm{t}, 2 \mathrm{H}), 1.84(\mathrm{~m}, 2 \mathrm{H})$, 1.47 (m, $2 \mathrm{H}), 0.94$ (t, $3 \mathrm{H}) .{ }^{13} \mathrm{C}$ NMR $\left(75 \mathrm{MHz}, \mathrm{CDCl}_{3}\right) \delta: 161.7,148.0,129.1,126.1,114.3$, 55.5, 51.0, 25.3, 21.6, 13.6. HRMS (ESI) $\mathrm{m} / \mathrm{z}$ calcd for $\mathrm{C}_{12} \mathrm{H}_{18} \mathrm{~N}_{2} \mathrm{O}_{3} \mathrm{~S}\left(\mathrm{M}^{+}\right)$270.1938, found $293.0944\left([\mathrm{M}+\mathrm{Na}]^{+}\right) ; 271.1110\left([\mathrm{M}+\mathrm{H}]^{+}\right)$.

\section{N'-(4-nitrobenzylidene) butane-1- sulfonyl hydrazone (3)}

Yellow powder, 83\% yield $(0.237 \mathrm{~g}) .{ }^{1} \mathrm{H}$ NMR $\left(300 \mathrm{MHz}, \mathrm{C}_{3} \mathrm{D}_{6} \mathrm{O}\right) \delta: 8.30(\mathrm{~d}, J=1.98 \mathrm{~Hz}, 2 \mathrm{H})$, $8.21(\mathrm{~s}, 1 \mathrm{H}), 7.97(\mathrm{~d}, J=5.04 \mathrm{~Hz}, 1 \mathrm{H}), 3.31(\mathrm{t}, 2 \mathrm{H}), 1.78(\mathrm{q}, 2 \mathrm{H}), 1.47(\mathrm{~m}, 2 \mathrm{H}), 0.91(\mathrm{t}, 3 \mathrm{H})$. ${ }^{13} \mathrm{C}$ NMR $\left(75 \mathrm{MHz}, \mathrm{CD}_{3} \mathrm{OD}\right) \delta: 149.8,145.0,141.5,128.8,124.9,49.2,26.6,21.0,14.0$. HRMS (ESI) $\mathrm{m} / z$ calcd for $\mathrm{C}_{11} \mathrm{H}_{15} \mathrm{~N}_{3} \mathrm{O}_{4} \mathrm{~S}\left(\mathrm{M}^{+}\right)$285.0783, found $308.0672\left([\mathrm{M}+\mathrm{Na}]^{+}\right) ; 286.0850$ $\left([\mathrm{M}+\mathrm{H}]^{+}\right)$. m.p. $136 \cdot 0-136.7^{\circ} \mathrm{C}$.

\section{N'-(4-hydroxy-3-methoxybenzylidene) butane-1- sulfonyl hydrazone (4)}

Yellow resin, 99\% yield $(0.284 \mathrm{~g}) .{ }^{1} \mathrm{H}$ NMR $\left(300 \mathrm{MHz}, \mathrm{CDCl}_{3}\right) \delta: 8.13(\mathrm{~s}, 1 \mathrm{H}), 7.78(\mathrm{~s}, 1 \mathrm{H})$, $7.29(\mathrm{~s}, 1 \mathrm{H}), 7.02(\mathrm{~d}, J=7.22 \mathrm{~Hz}, 1 \mathrm{H}), 6.89(\mathrm{~d}, J=7.99,1 \mathrm{H}), 5.95(\mathrm{~s}, 1 \mathrm{H}), 3.92(\mathrm{~s}, 3 \mathrm{H}), 3.30$ (t, $2 \mathrm{H}), 1.84$ (t, $2 \mathrm{H}), 1.47$ (q, $2 \mathrm{H}), 0.94$ (t, $3 \mathrm{H}) .{ }^{13} \mathrm{C} N M R\left(75 \mathrm{MHz}, \mathrm{CDCl}_{3}\right) \delta: 148.3,148.2$, $147.0,125.7,123.0,114.3,107.9,56.1,50.9,25.1,21.5,13.5$. HRMS (ESI) $\mathrm{m} / \mathrm{z}$ calcd for $\mathrm{C}_{12} \mathrm{H}_{18} \mathrm{~N}_{2} \mathrm{O}_{4} \mathrm{~S}\left(\mathrm{M}^{+}\right)$286.0987, found $309.0889\left([\mathrm{M}+\mathrm{Na}]^{+}\right) ; 287.1059\left([\mathrm{M}+\mathrm{H}]^{+}\right)$.

\section{N'-(thiophen-2-ylmethylene) butane-1- sulfonyl hydrazone (5)}

Dark-red powder, 99\% yield (0.244 g). ${ }^{1} \mathrm{H}$ NMR $\left(300 \mathrm{MHz}, \mathrm{CDCl}_{3}\right) \delta: 8.04(\mathrm{~s}, 1 \mathrm{H}), 7.82(\mathrm{~s}, 1$ H), $7.39(\mathrm{~d}, J=5.00 \mathrm{~Hz}, 1 \mathrm{H}), 7.27(\mathrm{~d}, J=4.17 \mathrm{~Hz}, 1 \mathrm{H}), 7.05\left(\mathrm{dd}, J^{1}=3.71 \mathrm{~Hz}, J^{2}=4.97 \mathrm{~Hz}, 1\right.$ H), $3.28(\mathrm{t}, 2 \mathrm{H}), 1.84(\mathrm{~m}, 1.90-1.80,2 \mathrm{H}), 1.46(\mathrm{~m}, 1.54-1.42,2 \mathrm{H}), 0.95(\mathrm{t}, 3 \mathrm{H}) .{ }^{13} \mathrm{C} \mathrm{NMR}(75$ $\mathrm{MHz}, \mathrm{CDCl}_{3}$ ) $\delta: 143.1,137.9,130.6,129.1,127.6,51.0,25.3,21.6,13.6$. HRMS (ESI) $\mathrm{m} / \mathrm{z}$ 
calcd for $\mathrm{C}_{9} \mathrm{H}_{14} \mathrm{~N}_{2} \mathrm{O}_{2} \mathrm{~S}_{2}\left(\mathrm{M}^{+}\right)$246.0497, found $269.0402\left([\mathrm{M}+\mathrm{Na}]^{+}\right) ; 247.0577\left([\mathrm{M}+\mathrm{H}]^{+}\right)$. m.p. 54.4-55. $5^{\circ} \mathrm{C}$.

\section{N'-benzylidene-ethane-sulfonyl hydrazone (6)}

Pale yellow powder, 95\% yield (0.202 g). ${ }^{1} \mathrm{H}$ NMR $\left(300 \mathrm{MHz}, \mathrm{CDCl}_{3}\right) \delta: 8.05(\mathrm{~s}, 1 \mathrm{H}), 7.85$ (s, $1 \mathrm{H}), 7.39\left(\mathrm{dd}, J^{1}=2.98 \mathrm{~Hz}, J^{2}=6.47 \mathrm{~Hz}, 2 \mathrm{H}\right), 7.39(\mathrm{~m}, 7.40-7.38,3 \mathrm{H}), 3.35(\mathrm{q}, J=7.41 \mathrm{~Hz}, 2$ H), $1.42(\mathrm{t}, 3 \mathrm{H}) .{ }^{13} \mathrm{C} \mathrm{NMR}\left(75 \mathrm{MHz}, \mathrm{CDCl}_{3}\right) \delta: 147.2,133.2,130.7,128.9,127.5,45.8,8.1$. HRMS (ESI) $\mathrm{m} / z$ calcd for $\mathrm{C}_{9} \mathrm{H}_{12} \mathrm{~N}_{2} \mathrm{O}_{2} \mathrm{~S}\left(\mathrm{M}^{+}\right)$212.0619, found $235.0514\left([\mathrm{M}+\mathrm{Na}]^{+}\right) ; 213.0690$ $\left([\mathrm{M}+\mathrm{H}]^{+}\right)$. m.p. $79 \cdot 6-80.5^{\circ} \mathrm{C}$.

\section{N'-(4-methoxybenzylidene) ethane-sulfonyl hydrazone (7)}

Dark yellow powder, 99\% yield (0.240 g). ${ }^{1} \mathrm{H}$ NMR (300 MHz, $\left.\mathrm{CDCl}_{3}\right) \delta: 8.39(\mathrm{~s}, 1 \mathrm{H}), 7.83(\mathrm{~s}$, $1 \mathrm{H}), 7.57(\mathrm{~d}, J=8.2 \mathrm{~Hz}, 2 \mathrm{H}), 6.87(\mathrm{~d}, J=8.2 \mathrm{~Hz}, 2 \mathrm{H}), 3.81(\mathrm{~s}, 3 \mathrm{H}), 3.32(\mathrm{q}, J=7.3 \mathrm{~Hz}, 2 \mathrm{H})$, 1.39 (t, $3 \mathrm{H}) .{ }^{13} \mathrm{C} \mathrm{NMR}\left(75 \mathrm{MHz}, \mathrm{CDCl}_{3}\right) \delta: 161.6,148.1,129.1,126.1,114.2,55.4,45.6,8.0$. HRMS (ESI) $m / z$ calcd for $\mathrm{C}_{10} \mathrm{H}_{14} \mathrm{~N}_{2} \mathrm{O}_{3} \mathrm{~S}\left(\mathrm{M}^{+}\right)$242.0725, found $265.0619\left([\mathrm{M}+\mathrm{Na}]^{+}\right) ; 243.0789$ $\left([\mathrm{M}+\mathrm{H}]^{+}\right)$. m.p. $110.7-111.2^{\circ} \mathrm{C}$.

\section{N'-(4-nitrobenzylidene) ethane-sulfonyl hydrazone (8)}

Yellow powder, $99 \%$ yield $(0.256 \mathrm{~g}) .{ }^{1} \mathrm{H}$ NMR $\left(300 \mathrm{MHz}, \mathrm{C}_{3} \mathrm{D}_{6} \mathrm{O}\right) \delta: 8.30(\mathrm{~d}, J=8.7 \mathrm{~Hz}, 2 \mathrm{H})$, $8.23(\mathrm{~s}, 1 \mathrm{H}), 7.97(\mathrm{~d}, J=8.8 \mathrm{~Hz}, 2 \mathrm{H}), 3.33(\mathrm{q}, 2 \mathrm{H}), 1.33(\mathrm{t}, 3 \mathrm{H}) .{ }^{13} \mathrm{C} \mathrm{NMR}\left(75 \mathrm{MHz}, \mathrm{CD}_{3} \mathrm{OD}\right)$ $\delta: 149.9,145.0,141.5,128.8,124.9,46.6$, 8.1. HRMS (ESI) $\mathrm{m} / \mathrm{z}$ calcd for $\mathrm{C}_{9} \mathrm{H}_{11} \mathrm{~N}_{3} \mathrm{O}_{4} \mathrm{~S}\left(\mathrm{M}^{+}\right)$ 257.0470 , found $280.0366\left([\mathrm{M}+\mathrm{Na}]^{+}\right) ; 258.0541\left([\mathrm{M}+\mathrm{H}]^{+}\right)$. m.p. $180.4-180.9^{\circ} \mathrm{C}$.

\section{N'-(4-hydroxy-3-methoxybenzylidene) ethanesulfonyl hydrazone (9)}

Yellow resin, 99\% yield $(0.257 \mathrm{~g}) .{ }^{1} \mathrm{H}$ NMR $\left(300 \mathrm{MHz},\left(\mathrm{CD}_{3}\right)_{2} \mathrm{SO}\right) \delta: 10.84(\mathrm{~s}, 1 \mathrm{H}), 9.46(\mathrm{~s}, 1$ H), $7.89(\mathrm{~s}, 1 \mathrm{H}), 7.20(\mathrm{~s}, 1 \mathrm{H}), 7.04(\mathrm{~d}, \mathrm{~J}=7.82 \mathrm{~Hz}, 1 \mathrm{H}), 6.81(\mathrm{~d}, \mathrm{~J}=7,95,1 \mathrm{H}), 3.80(\mathrm{~s}, 3 \mathrm{H})$, 3.20 (q, $2 \mathrm{H}), 1.21$ (t, $3 \mathrm{H}) .{ }^{13} \mathrm{C} \mathrm{NMR}\left(75 \mathrm{MHz},\left(\mathrm{CD}_{3}\right)_{2} \mathrm{SO}\right) \delta: 148.4,147.5,146.8,124.8,120.9$, 115.1, 109.0, 55.2, 44.0, 7.3. HRMS (ESI) $\mathrm{m} / z$ calcd for $\mathrm{C}_{10} \mathrm{H}_{14} \mathrm{~N}_{2} \mathrm{O}_{4} \mathrm{~S}\left(\mathrm{M}^{+}\right)$258.0674, found $281.0574\left([\mathrm{M}+\mathrm{Na}]^{+}\right) ; 259.0745\left([\mathrm{M}+\mathrm{H}]^{+}\right)$.

\section{$N^{\prime}$-benzylidenebenzenesulfonyl hydrazone (10)}

Yellow powder, $61 \%$ yield $(0.159 \mathrm{~g}) .{ }^{1} \mathrm{H}$ NMR $\left(300 \mathrm{MHz}, \mathrm{CDCl}_{3}\right) \delta: 8.23(\mathrm{~s}, 1 \mathrm{H}), 8.00(\mathrm{~d}, J=$ $7.05 \mathrm{~Hz}, 2 \mathrm{H}), 7.78(\mathrm{~s}, 1 \mathrm{H}), 7.61-7.49(\mathrm{~m}, 5 \mathrm{H}), 7.36-7.33(\mathrm{~m}, 3 \mathrm{H}) .{ }^{13} \mathrm{C} \mathrm{NMR}\left(75 \mathrm{MHz}, \mathrm{CDCl}_{3}\right)$ $\delta: 148.2,138.4,133.5,133.3,130.6,129.2,128.8,128.0,127.5$. HRMS (ESI) $\mathrm{m} / \mathrm{z}$ calcd for $\mathrm{C}_{13} \mathrm{H}_{12} \mathrm{~N}_{2} \mathrm{O}_{2} \mathrm{~S}\left(\mathrm{M}^{+}\right) 260.0619$, found $283.0524\left([\mathrm{M}+\mathrm{Na}]^{+}\right) ; 261.0695\left([\mathrm{M}+\mathrm{H}]^{+}\right)$. m.p. 106.8$108.0^{\circ} \mathrm{C}$.

\section{N'-(4-methoxybenzylidene) benzenesulfonyl hydrazone (11)}

White powder, $54 \%$ yield $(0.157 \mathrm{~g}) .{ }^{1} \mathrm{H}$ NMR $\left(300 \mathrm{MHz}, \mathrm{CDCl}_{3}\right) \delta: 8.00(\mathrm{~d}, J=7.29 \mathrm{~Hz}, 2 \mathrm{H})$, $7.79(\mathrm{~s}, 1 \mathrm{H}), 7.73(\mathrm{~s}, 1 \mathrm{H}), 7.61-7.48(\mathrm{~m}, 5 \mathrm{H}), 6.87(\mathrm{~d}, J=8.67 \mathrm{~Hz}, 2 \mathrm{H}), 3.82(\mathrm{~s}, 3 \mathrm{H}) .{ }^{13} \mathrm{C}$ NMR $\left(75 \mathrm{MHz}, \mathrm{CDCl}_{3}\right) \delta: 161.8,148.6,138.6,133.4,129.1,128.0,128.1,126.0,114.3,55.5$. HRMS (ESI) $m / z$ calcd for $\mathrm{C}_{14} \mathrm{H}_{14} \mathrm{~N}_{2} \mathrm{O}_{3} \mathrm{~S}\left(\mathrm{M}^{+}\right)$290.0725, found 313.0625 ([M+Na] $\left.]^{+}\right) ; 291.0796$ $\left([\mathrm{M}+\mathrm{H}]^{+}\right)$. m.p. $151.9-152.2^{\circ} \mathrm{C}$.

\section{N'-(4-nitrobenzylidene) benzenesulfonyl hydrazone (12)}

Yellow powder, $95 \%$ yield $(0.290 \mathrm{~g}) .{ }^{1} \mathrm{H}$ NMR $\left(300 \mathrm{MHz}, \mathrm{C}_{3} \mathrm{D}_{6} \mathrm{O}\right) \delta: 8.25(\mathrm{~d}, J=8.6 \mathrm{~Hz}, 2 \mathrm{H})$, $8.13(\mathrm{~s}, 1 \mathrm{H}), 7.99(\mathrm{~d}, J=7.0 \mathrm{~Hz}, 2 \mathrm{H}), 7.89(\mathrm{~d}, J=8.6 \mathrm{~Hz}, 2 \mathrm{H}), 7.70-7.59(\mathrm{~m}, 3 \mathrm{H}) .{ }^{13} \mathrm{C} \mathrm{NMR}$ (75 MHz, CD $\mathrm{OD}$ ) $\delta: 149.8,145.6,141.3,140.4,134.3,130.2,128.8,128.7,124,9$. HRMS (ESI) $\mathrm{m} / \mathrm{z}$ calcd for $\mathrm{C}_{13} \mathrm{H}_{12} \mathrm{~N}_{3} \mathrm{O}_{4} \mathrm{~S}\left(\mathrm{M}^{+}\right) 305.0470$, found $328.0371\left([\mathrm{M}+\mathrm{Na}]^{+}\right) ; 306.0551$ $\left([\mathrm{M}+\mathrm{H}]^{+}\right)$. m.p. $150 \cdot 6-151 \cdot 3^{\circ} \mathrm{C}$ (ref. $\left.164-166^{1}\right)$. 


\section{N'-(4-hydroxy-3-methoxybenzylidene) benzenesulfonyl hydrazone (13)}

White powder, $34 \%$ yield $(0.104 \mathrm{~g}) .{ }^{1} \mathrm{H}$ NMR $\left(300 \mathrm{MHz}, \mathrm{CDCl}_{3}\right) \delta: 7.99\left(\mathrm{dd}, J^{1}=11.7 \mathrm{~Hz}, J^{2}=\right.$ $4.4 \mathrm{~Hz}, 2 \mathrm{H}), 7.71(\mathrm{~s}, 1 \mathrm{H}), 7.58-7.48(\mathrm{~m}, 3 \mathrm{H}), 7.21(\mathrm{~d}, J=1.5 \mathrm{~Hz}, 1 \mathrm{H}), 6.96\left(\mathrm{dd}, J^{1}=8.1 \mathrm{~Hz}, J^{2}\right.$ $=1.7 \mathrm{~Hz}, 1 \mathrm{H}), 6.85(\mathrm{~d}, J=8.1 \mathrm{~Hz}, 1 \mathrm{H}), 5.88(\mathrm{~s}, 1 \mathrm{H}), 3.90(\mathrm{~s}, 3 \mathrm{H}) .{ }^{13} \mathrm{C} \mathrm{NMR}\left(75 \mathrm{MHz}, \mathrm{CDCl}_{3}\right)$ $\delta: 149.0,148.4,147.1,138.6,133.4,129.2,128.0,125.8,123.1,114.4,108.1,56.2$. HRMS (ESI) $\mathrm{m} / \mathrm{z}$ calcd for $\mathrm{C}_{14} \mathrm{H}_{14} \mathrm{~N}_{2} \mathrm{O}_{4} \mathrm{~S}\left(\mathrm{M}^{+}\right)$306.0674, found $329.0582\left([\mathrm{M}+\mathrm{Na}]^{+}\right) ; 307.0751$ $\left([\mathrm{M}+\mathrm{H}]^{+}\right)$. m.p. $82 \cdot 2-82 \cdot 6^{\circ} \mathrm{C}$.

\section{4-nitro-benzaldehyde hydrazone (14)}

Yellow powder, 99\% yield $(0.202 \mathrm{~g}) .{ }^{1} \mathrm{H}$ NMR $\left(300 \mathrm{MHz},\left(\mathrm{CD}_{3}\right)_{2} \mathrm{SO}\right) \delta: 7.71\left(\mathrm{~d}, \mathrm{~J}^{1}=7.1 \mathrm{~Hz}, 2\right.$ H), $7.33(\mathrm{~s}, 1 \mathrm{H}), 7.23$ (d, J= 7.3 Hz, 1 H). m.p. $140.8-141.7^{\circ} \mathrm{C}$.

3. Copy of ${ }^{1} \mathrm{H}$ and ${ }^{13} \mathrm{C}$ NMR spectra of compounds 1 to 14
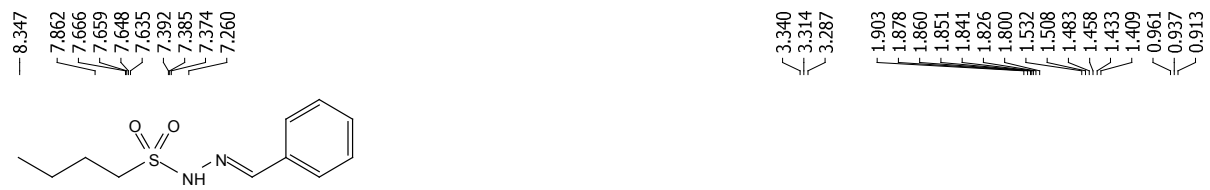

(1)

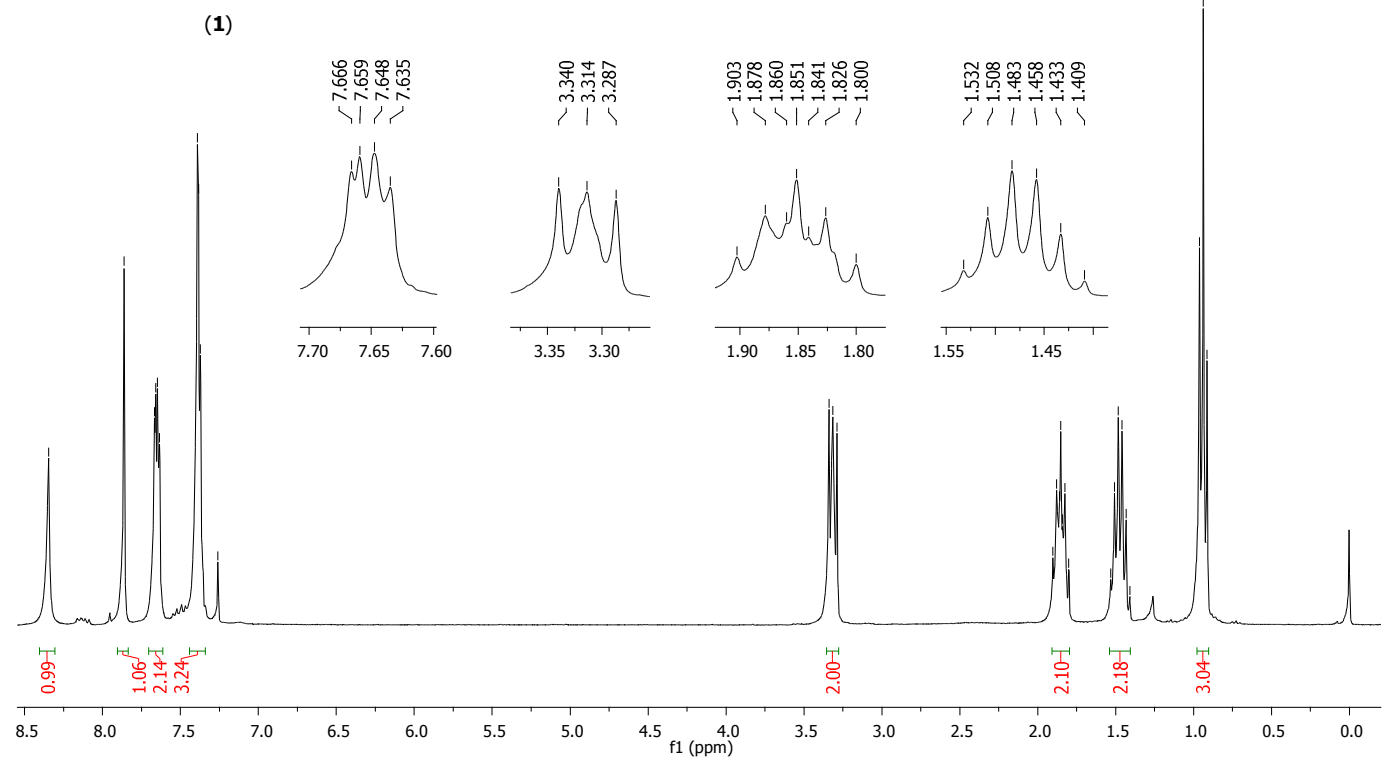



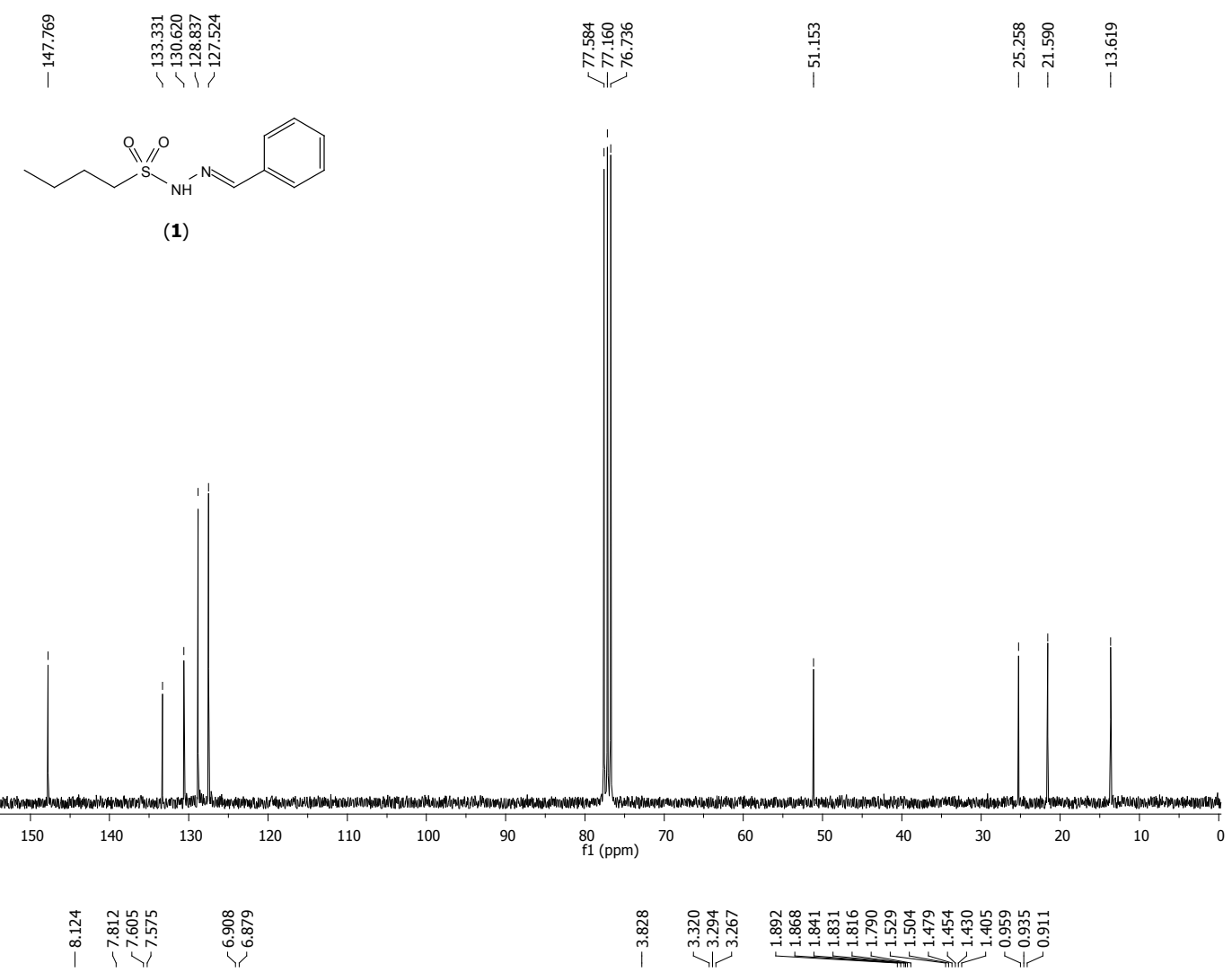

৷

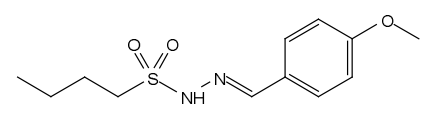

(2)
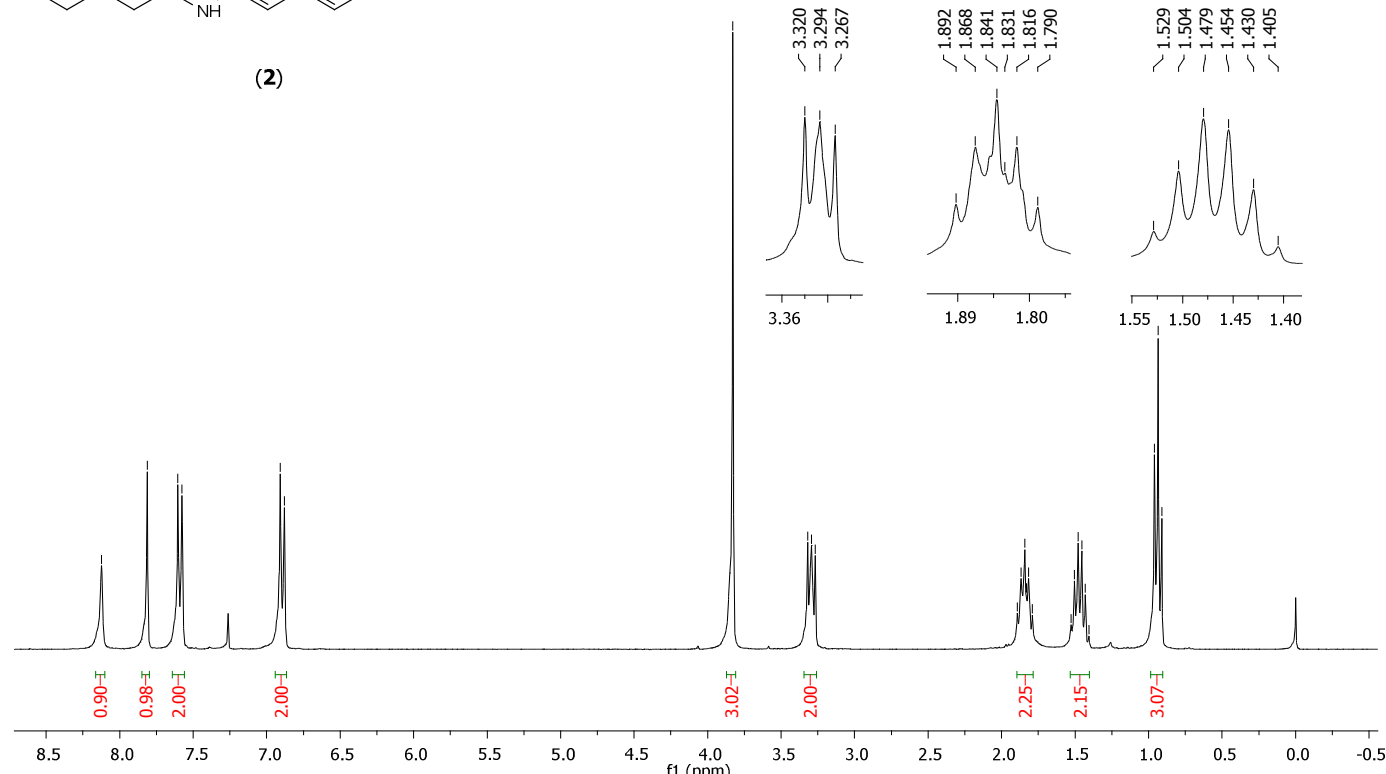


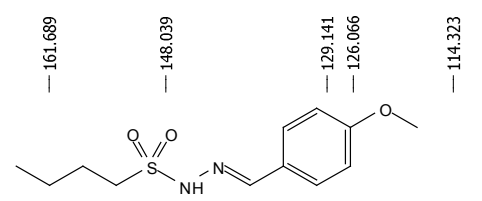

(2)
架是角

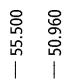

兽
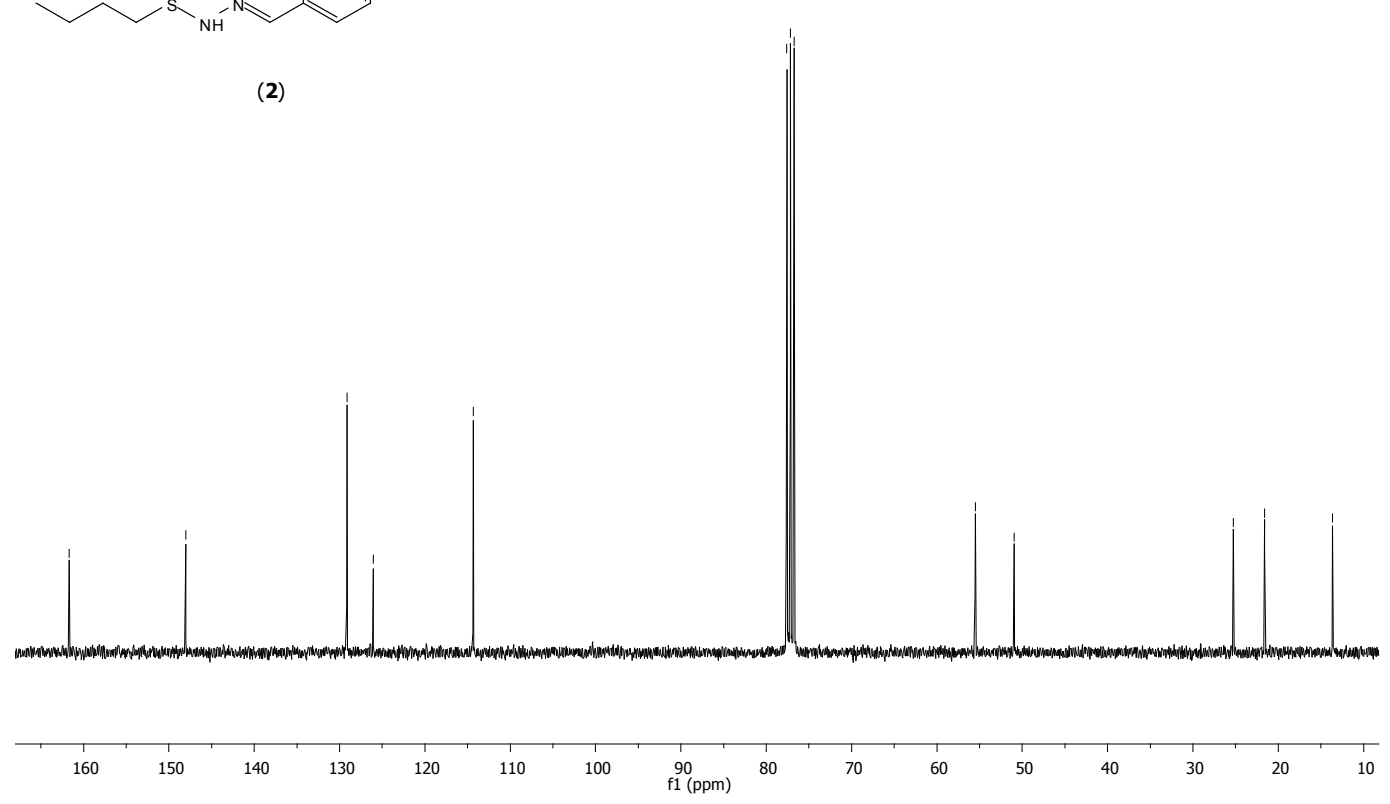

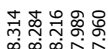

|
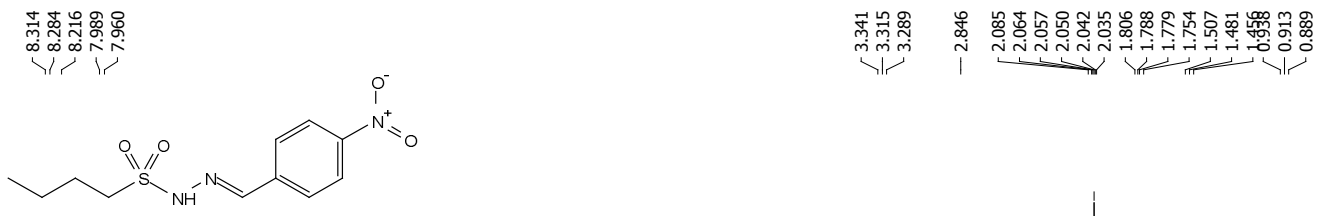

(3)

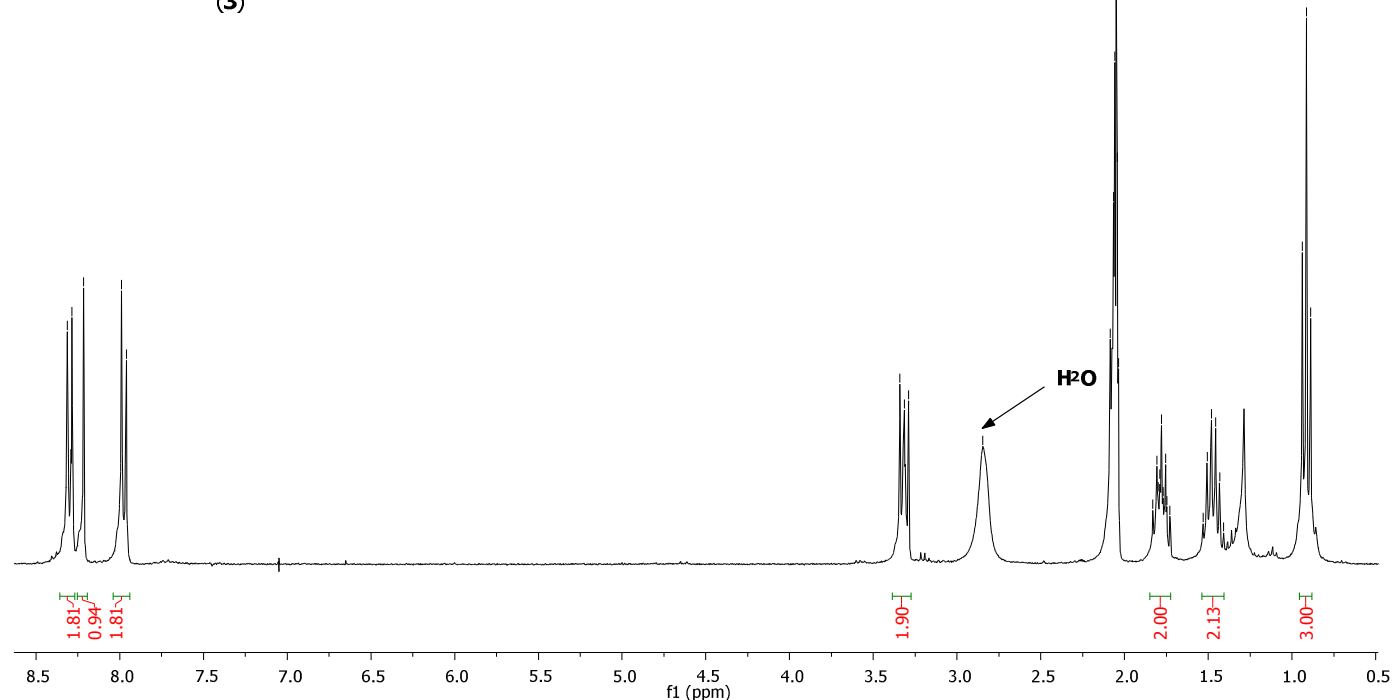



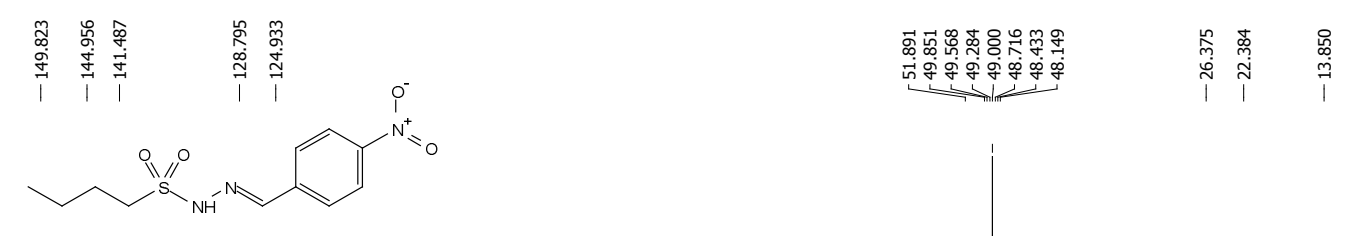

(3)
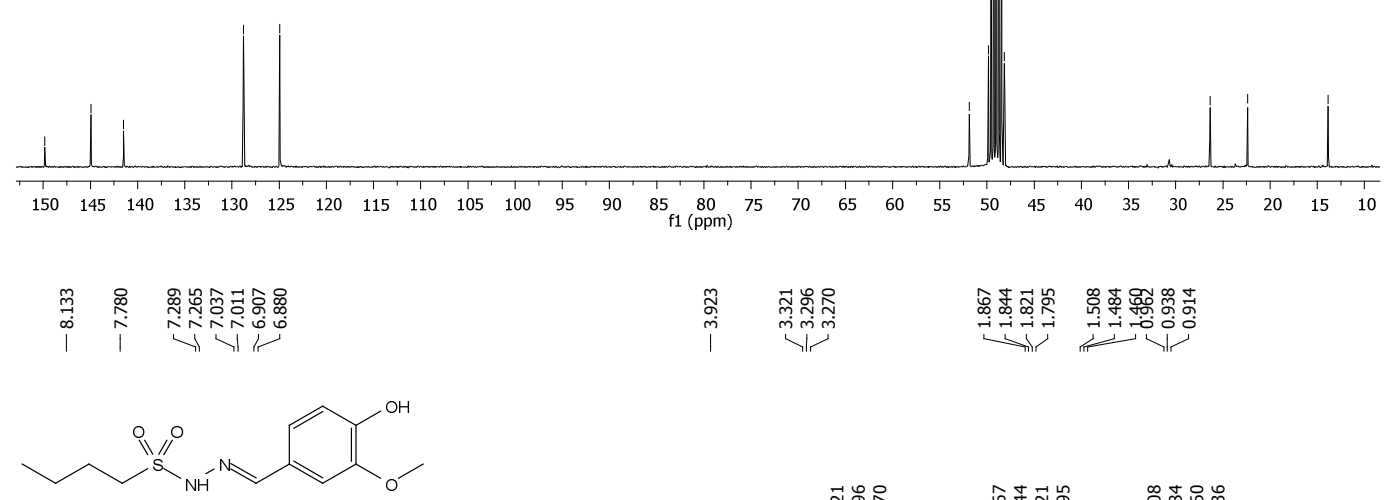

(4)
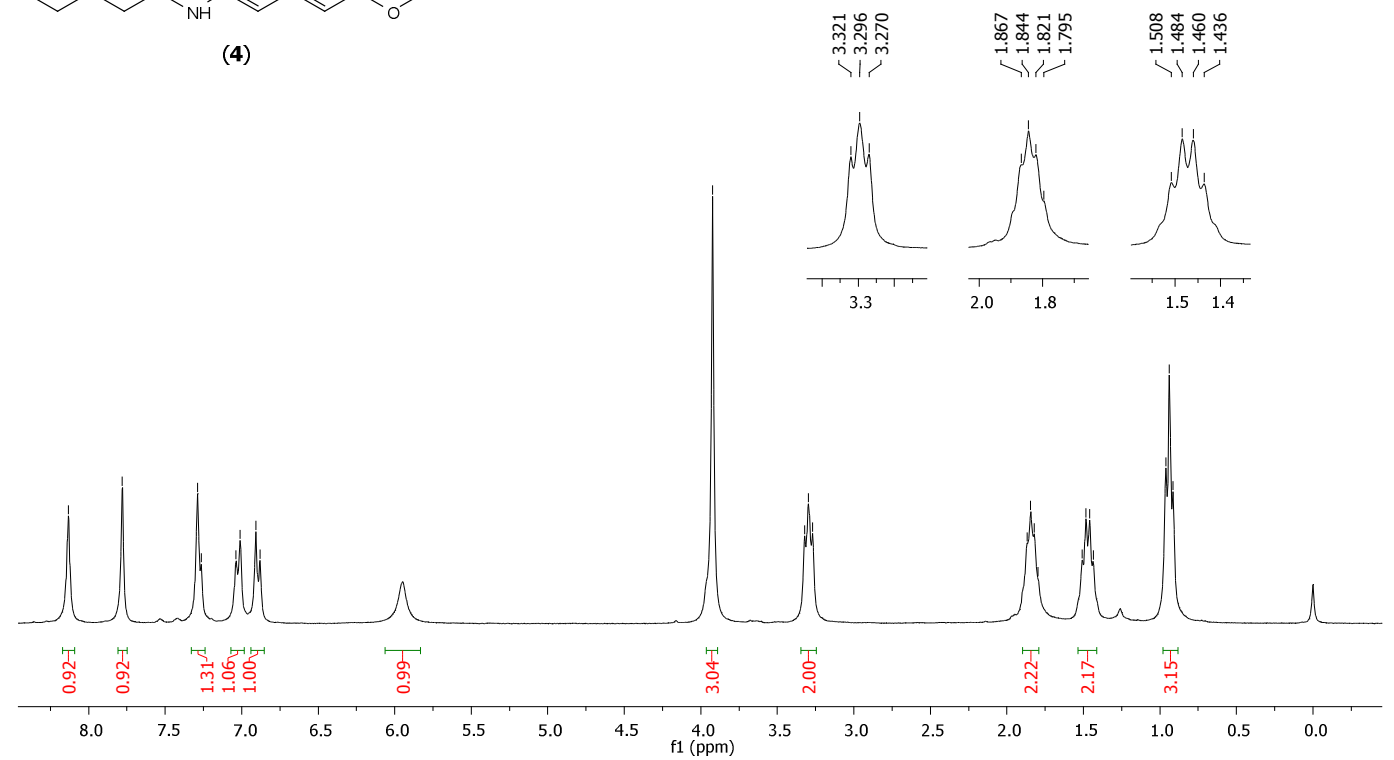

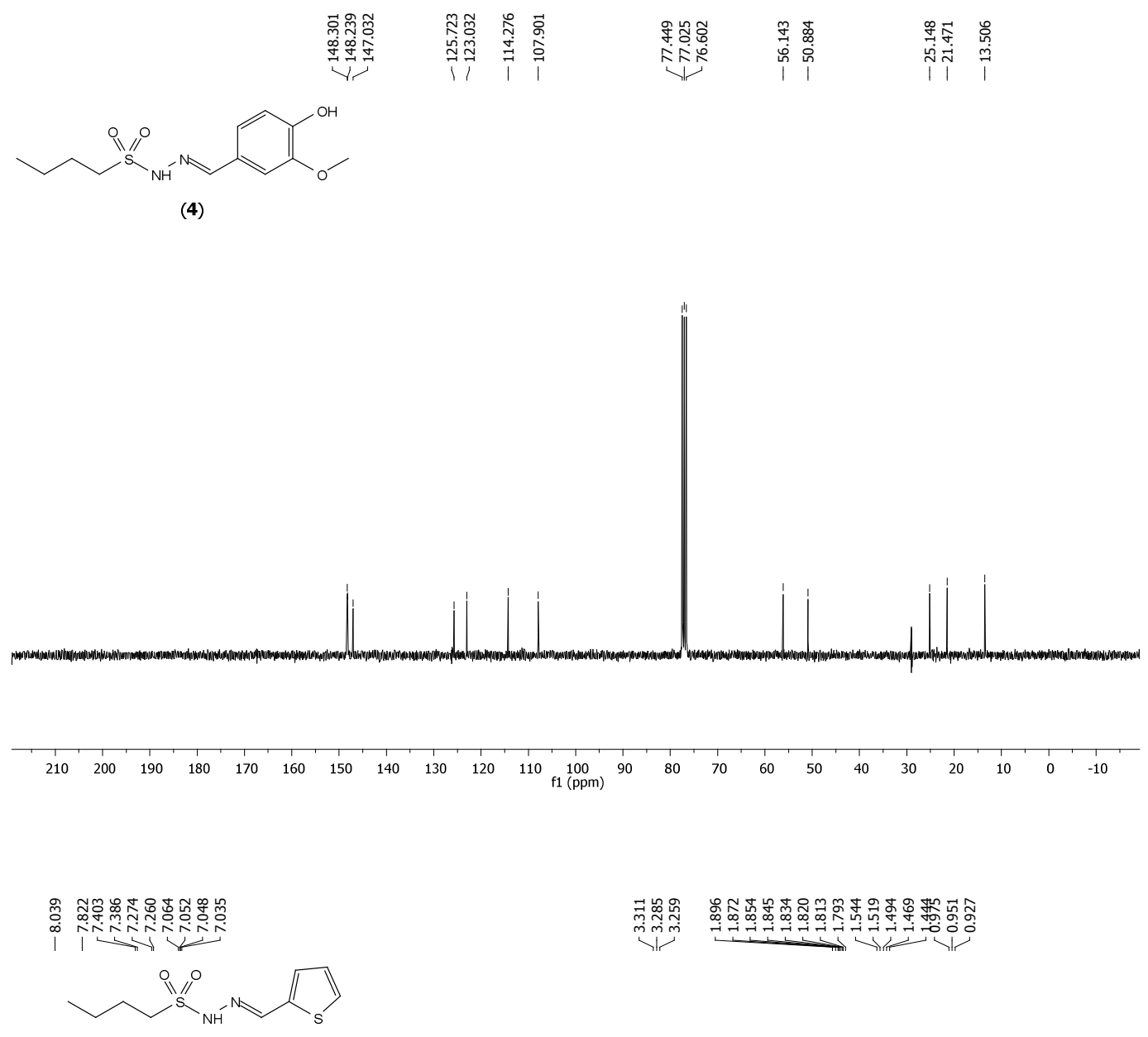

(5)

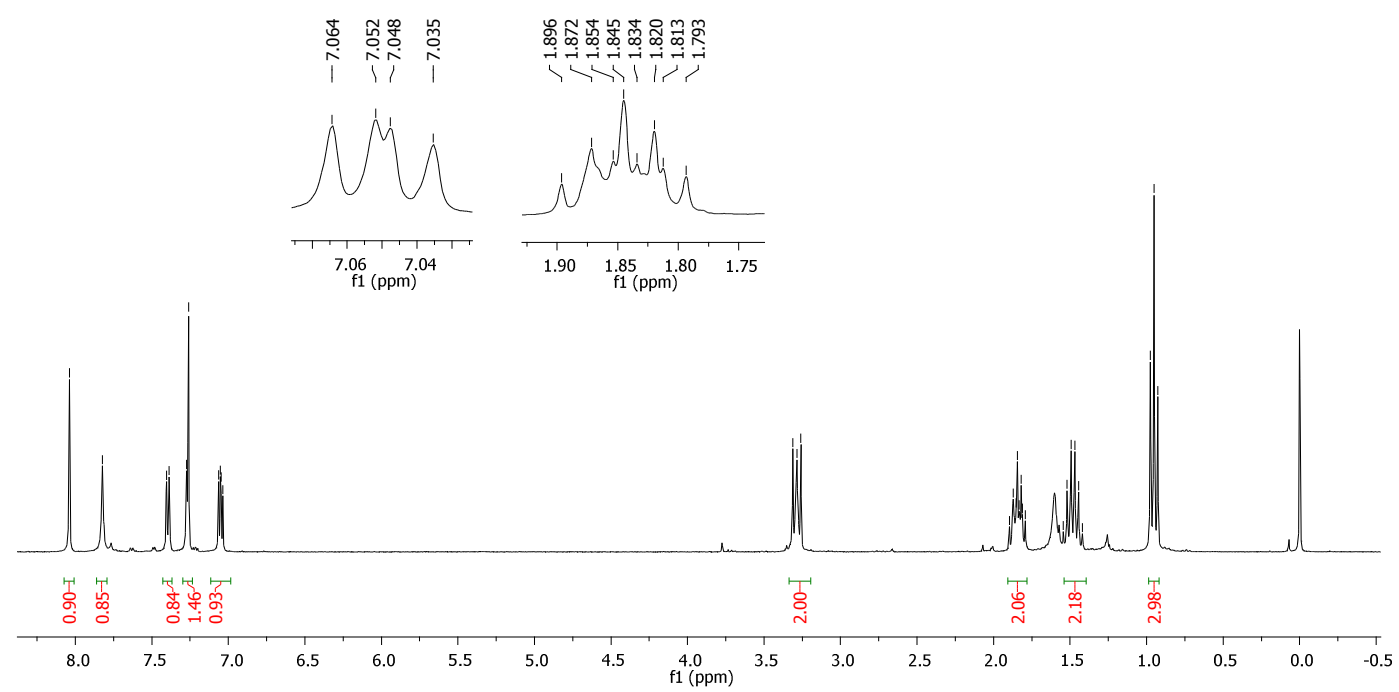




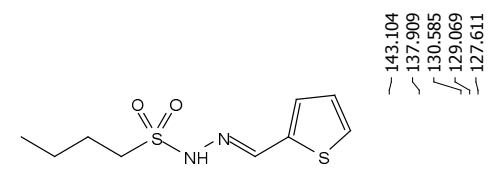

(5)
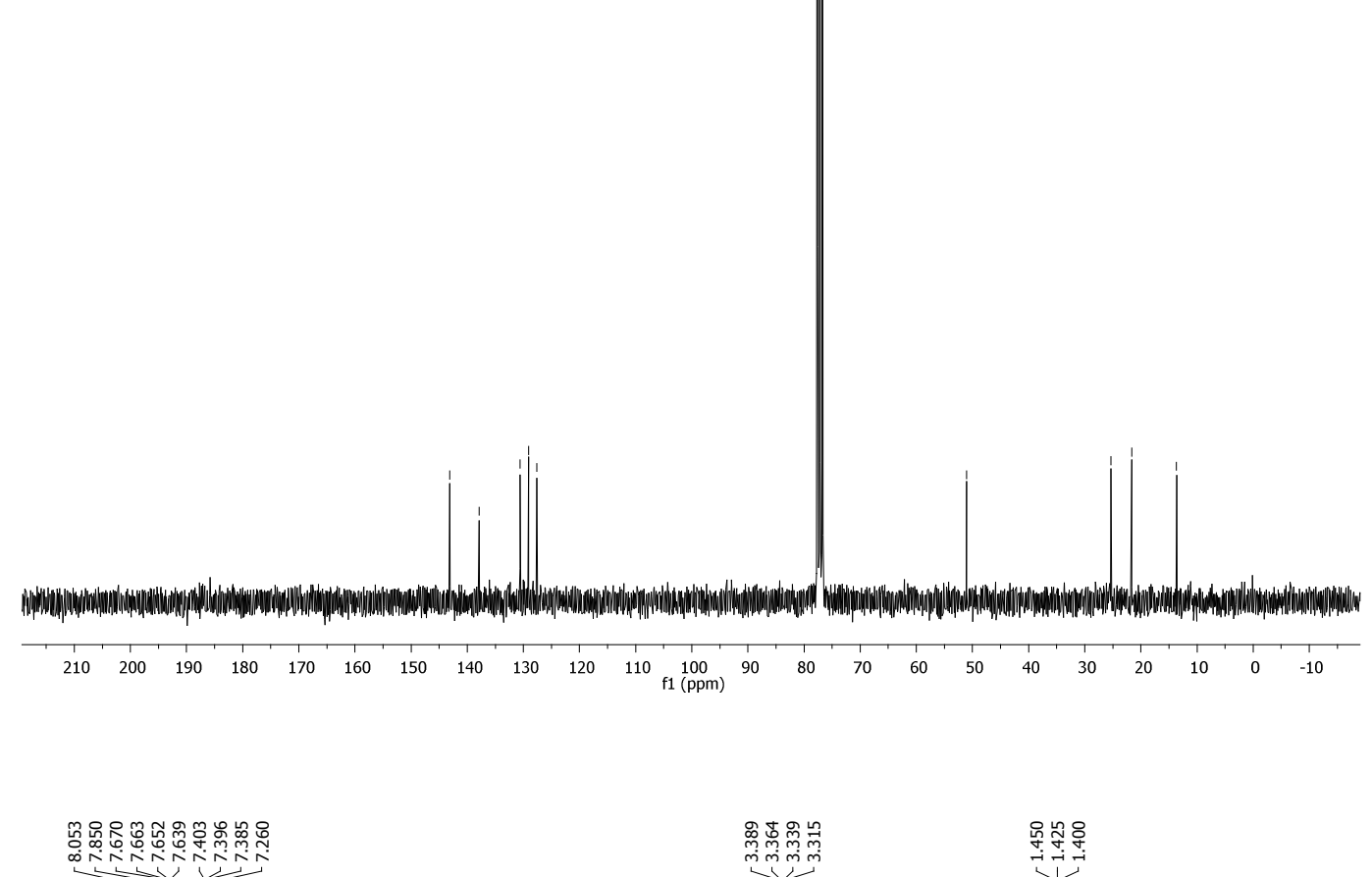

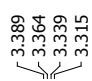

号过尔

$\mathbb{S}_{\mathrm{S}}^{\mathrm{O}}$

(6)

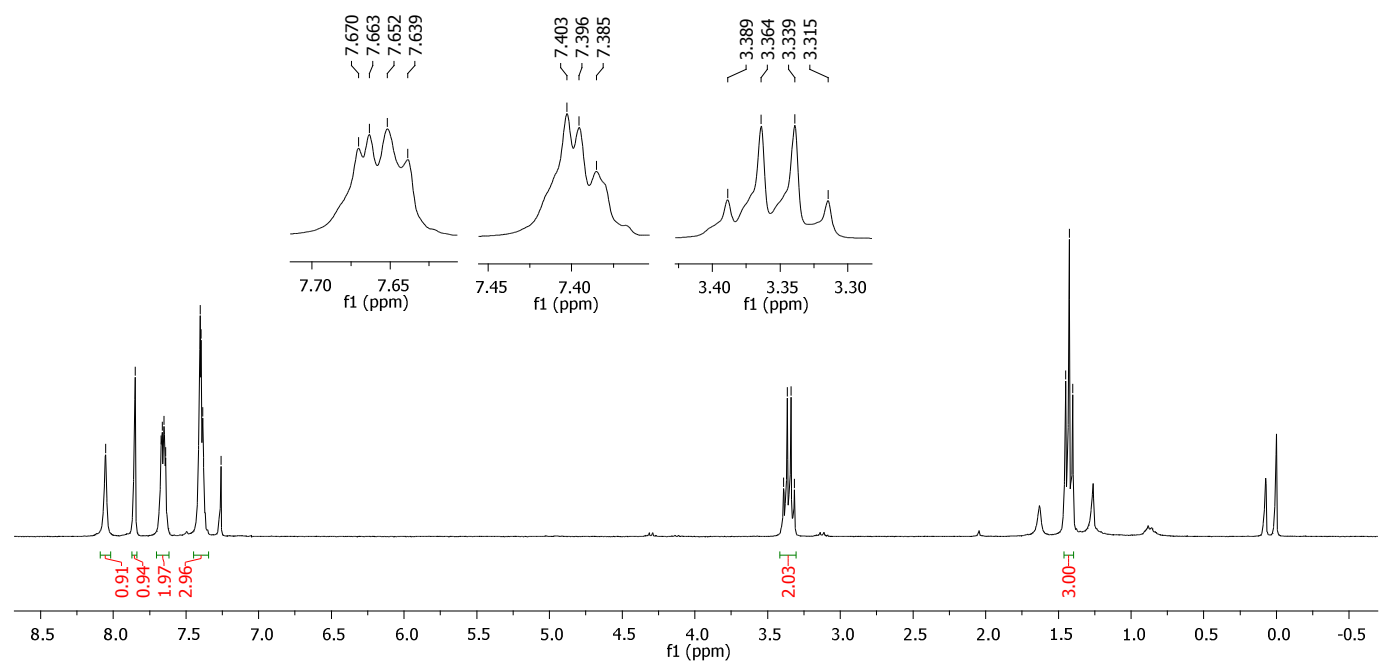




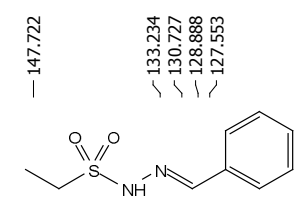

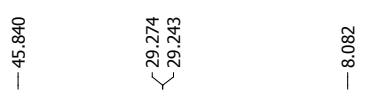

(6)

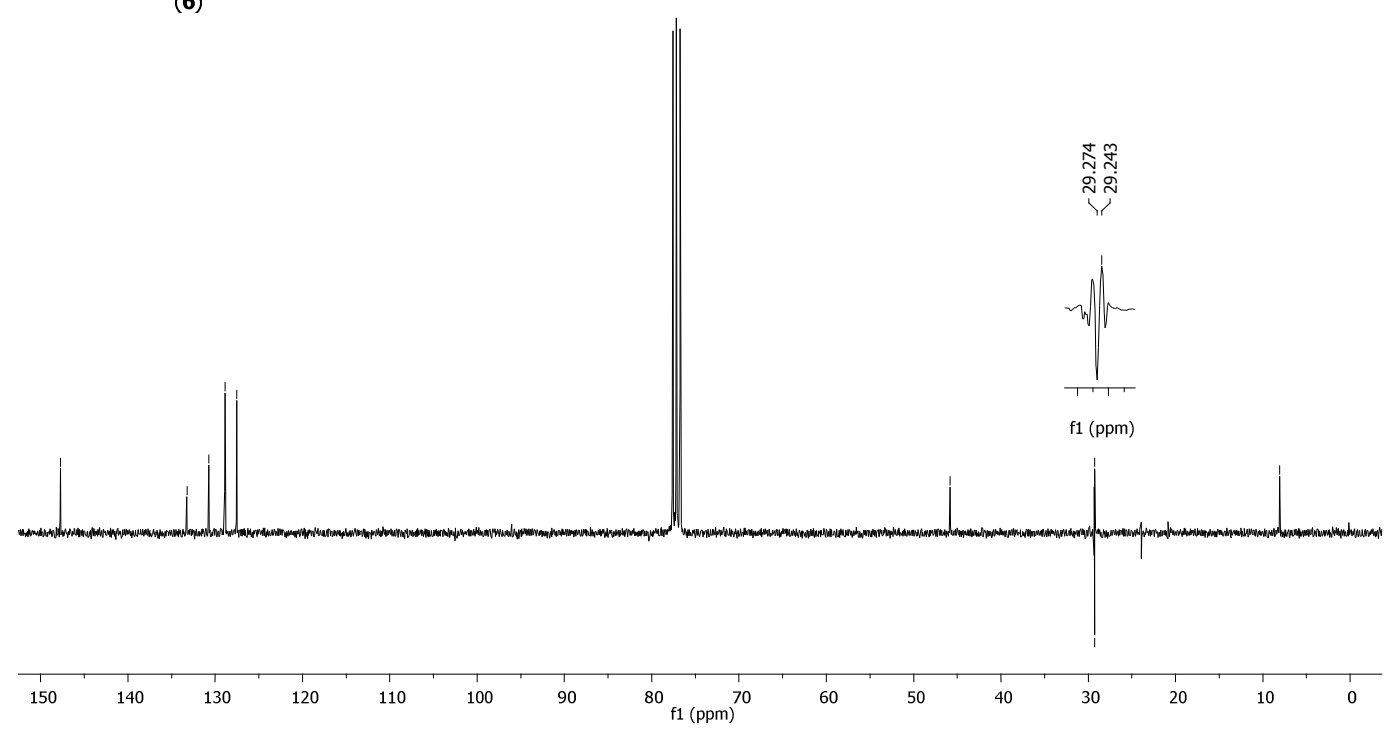

$\underbrace{1 / 1}_{N H}$

诺

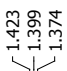

(7)

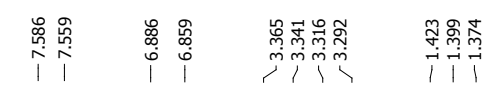
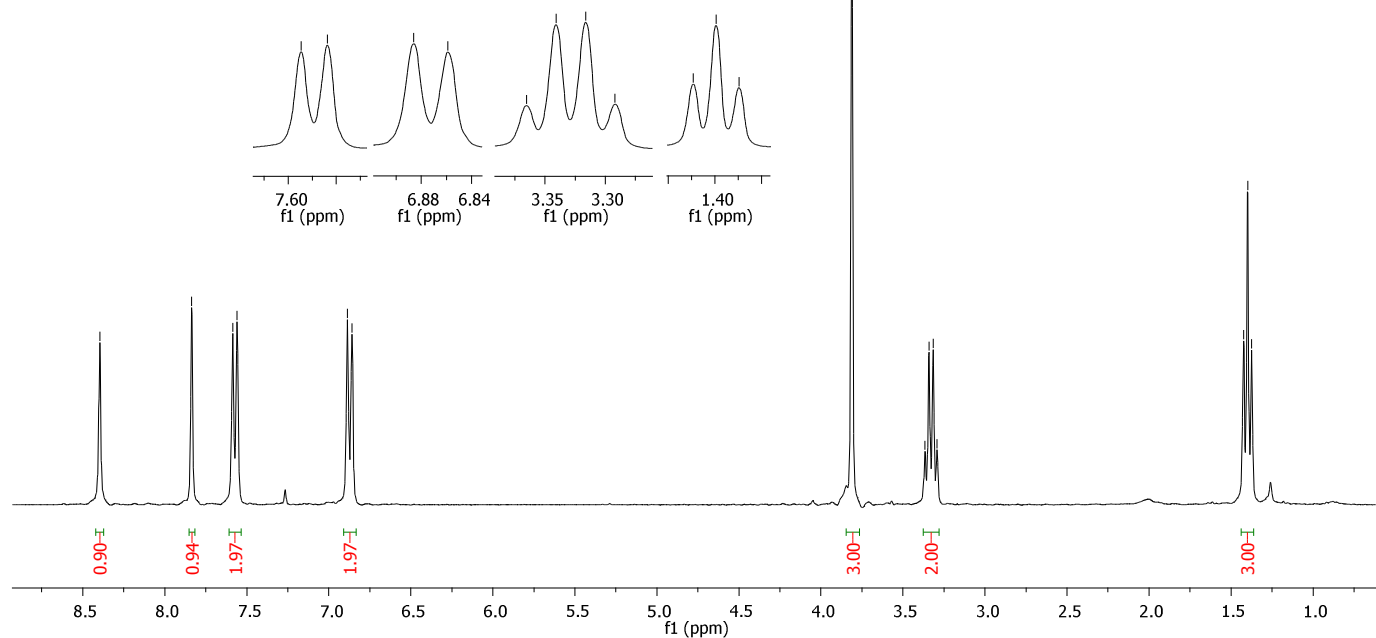

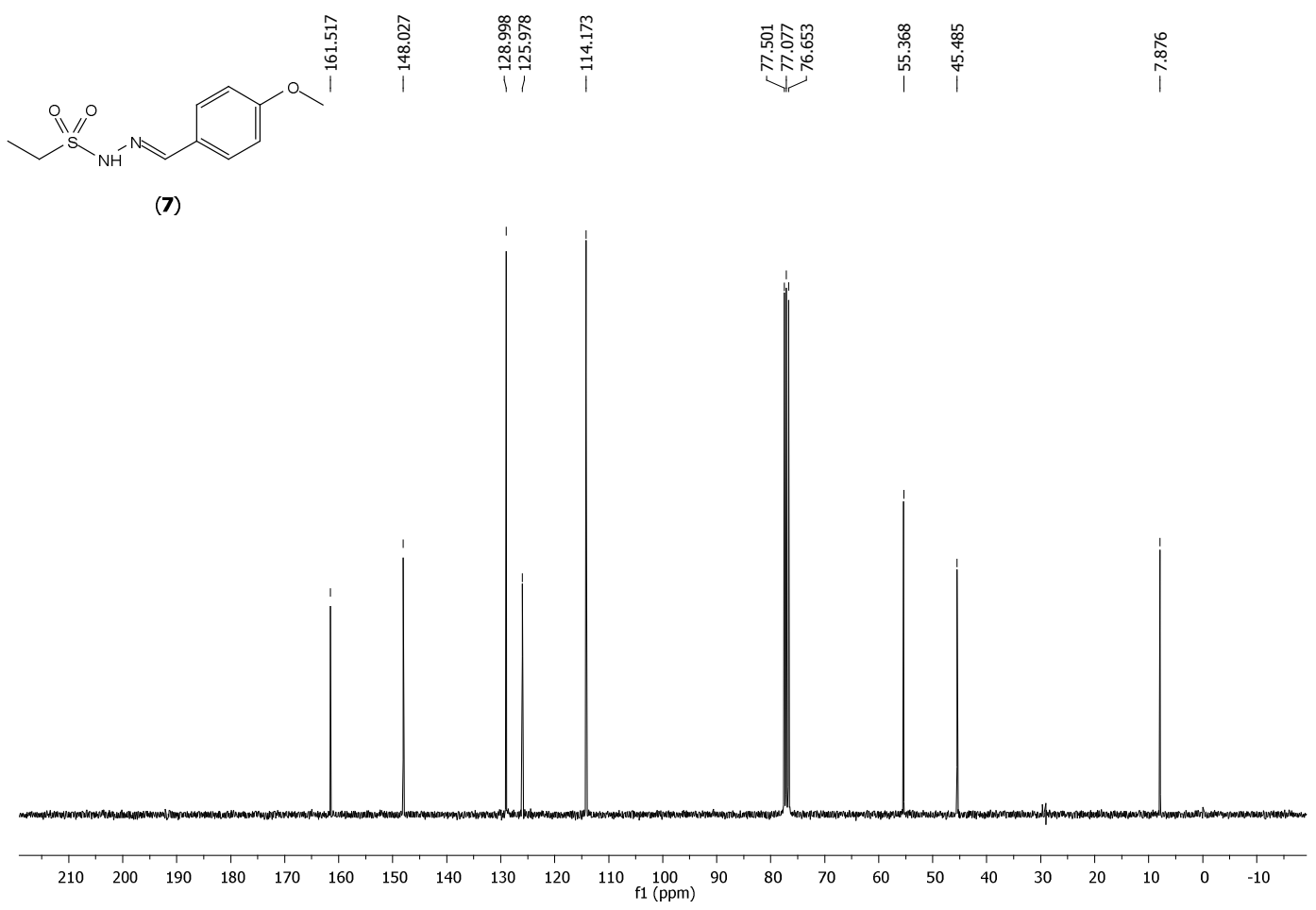

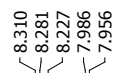

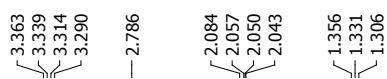

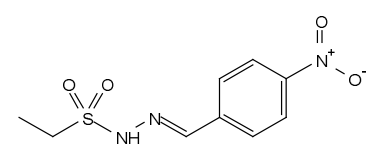

(8)

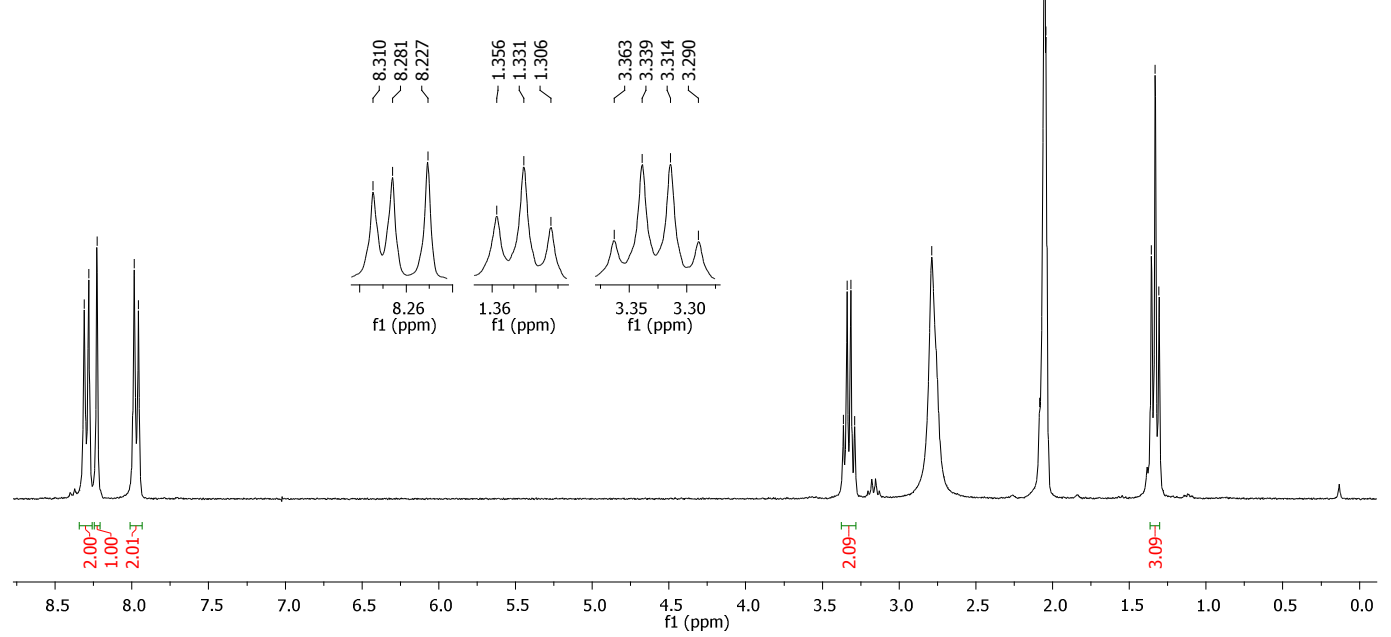




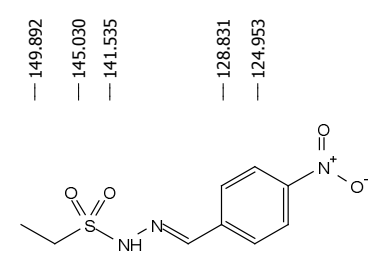

(8)
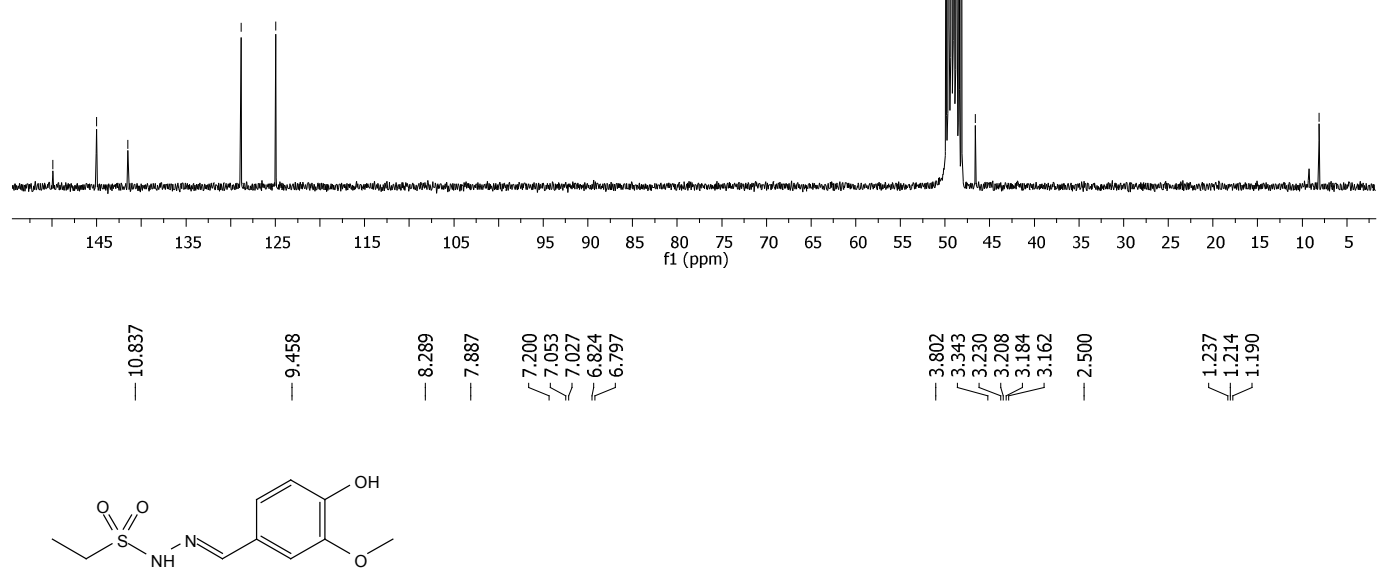

(9)
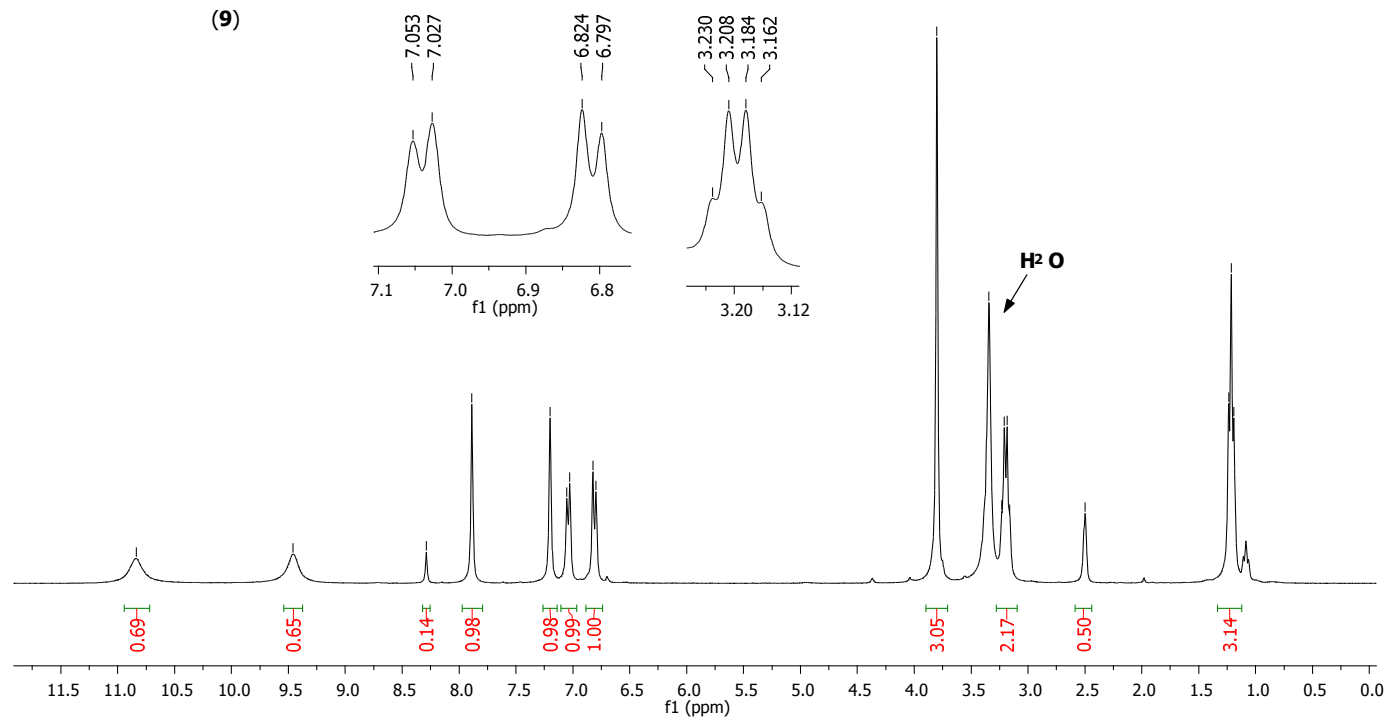


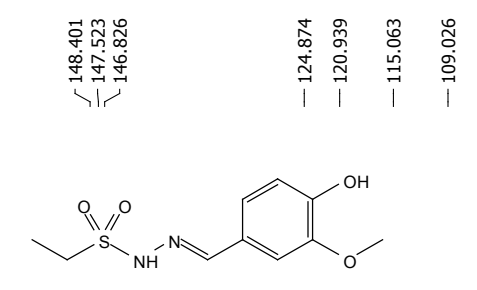

(9)
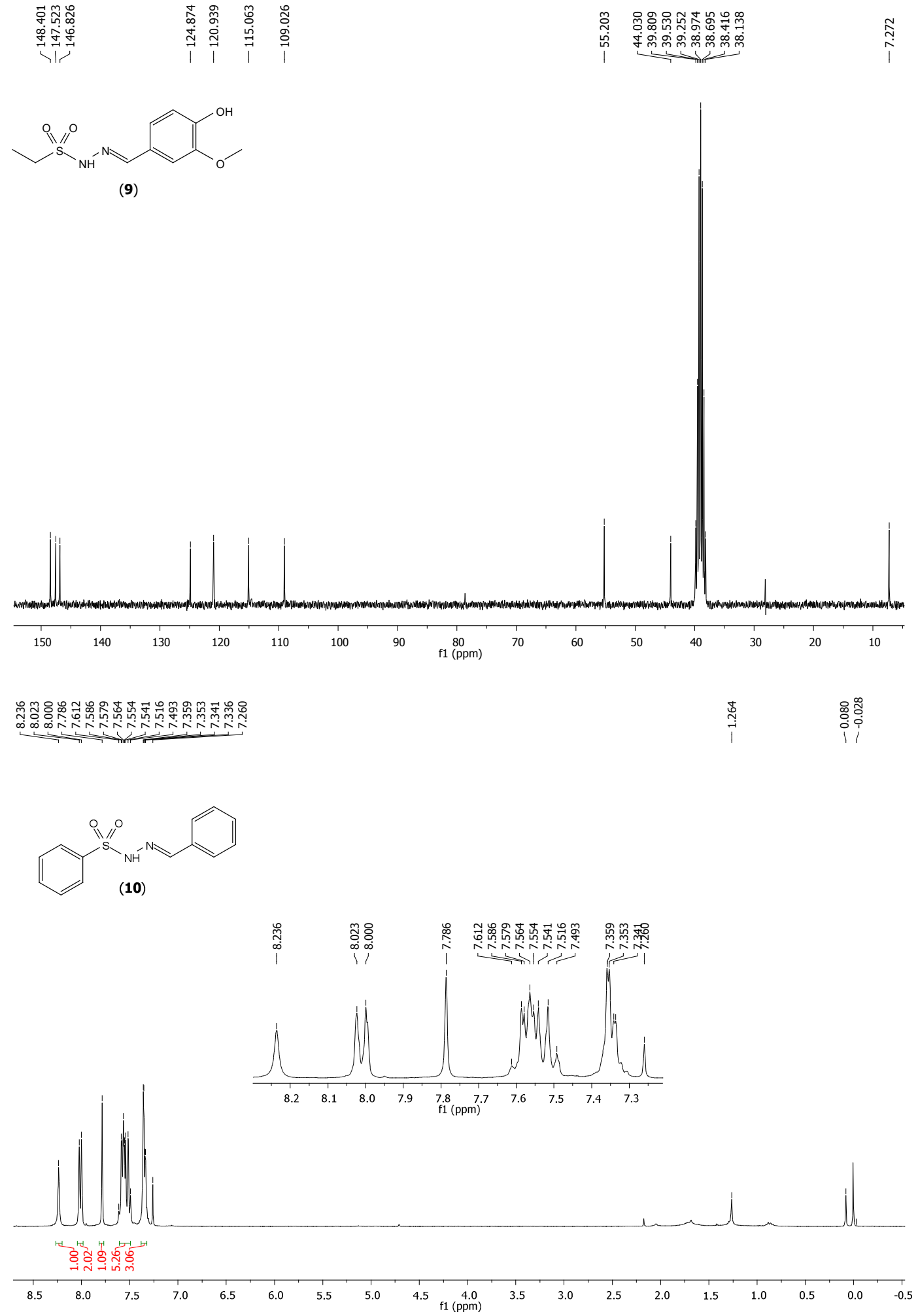


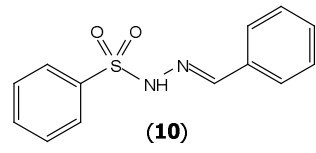

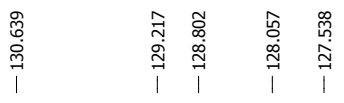
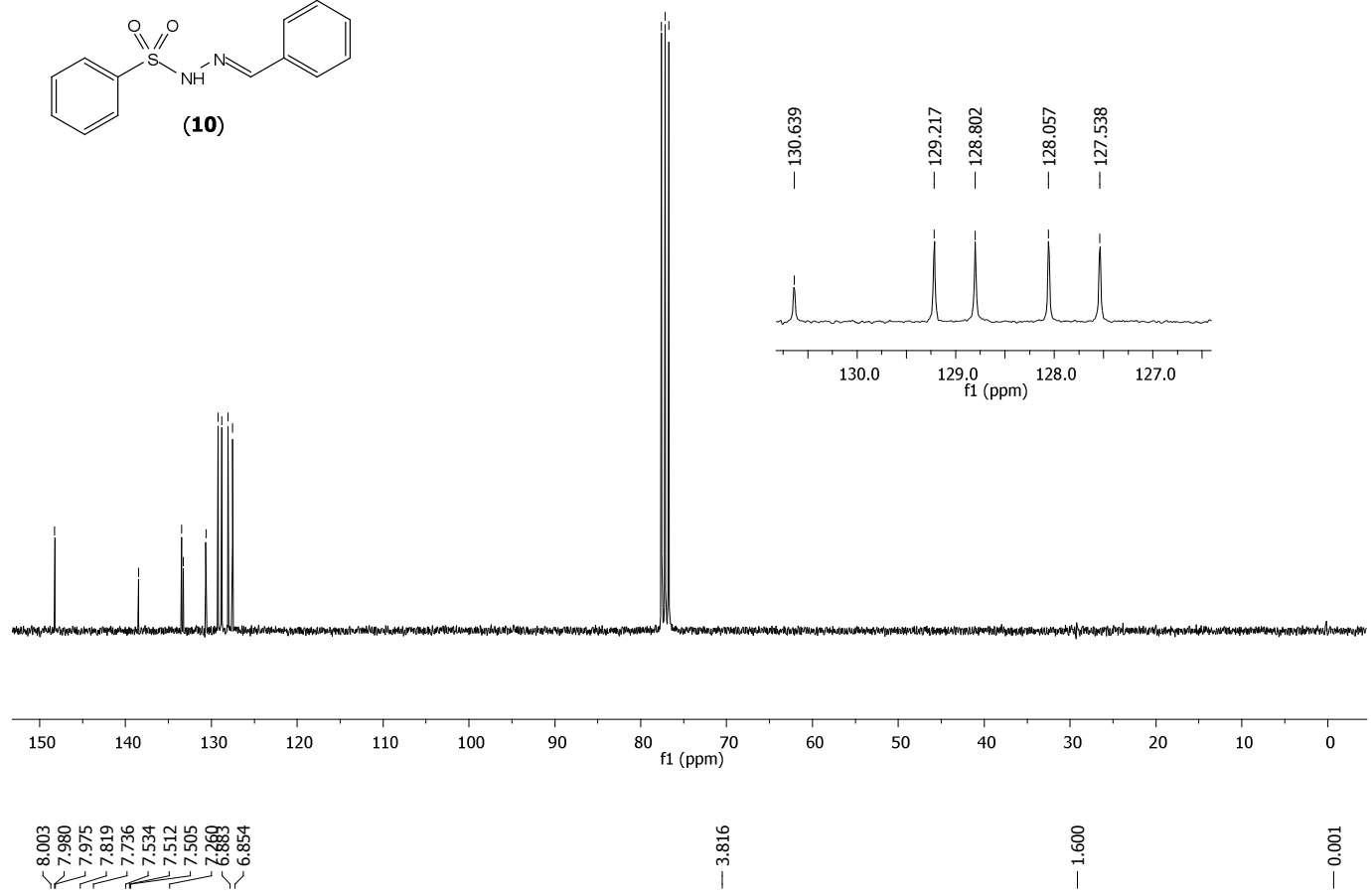<smiles>COc1ccc(C=CNS(=O)(=O)c2ccccc2)cc1</smiles>

(11)
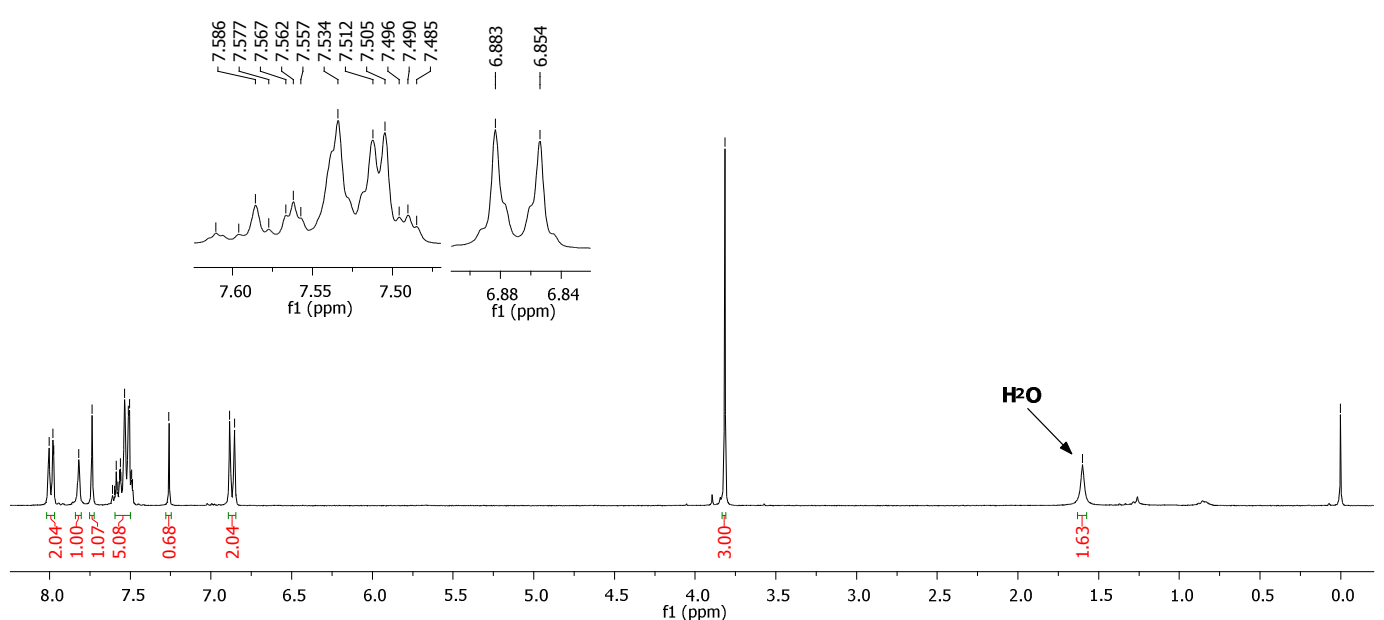


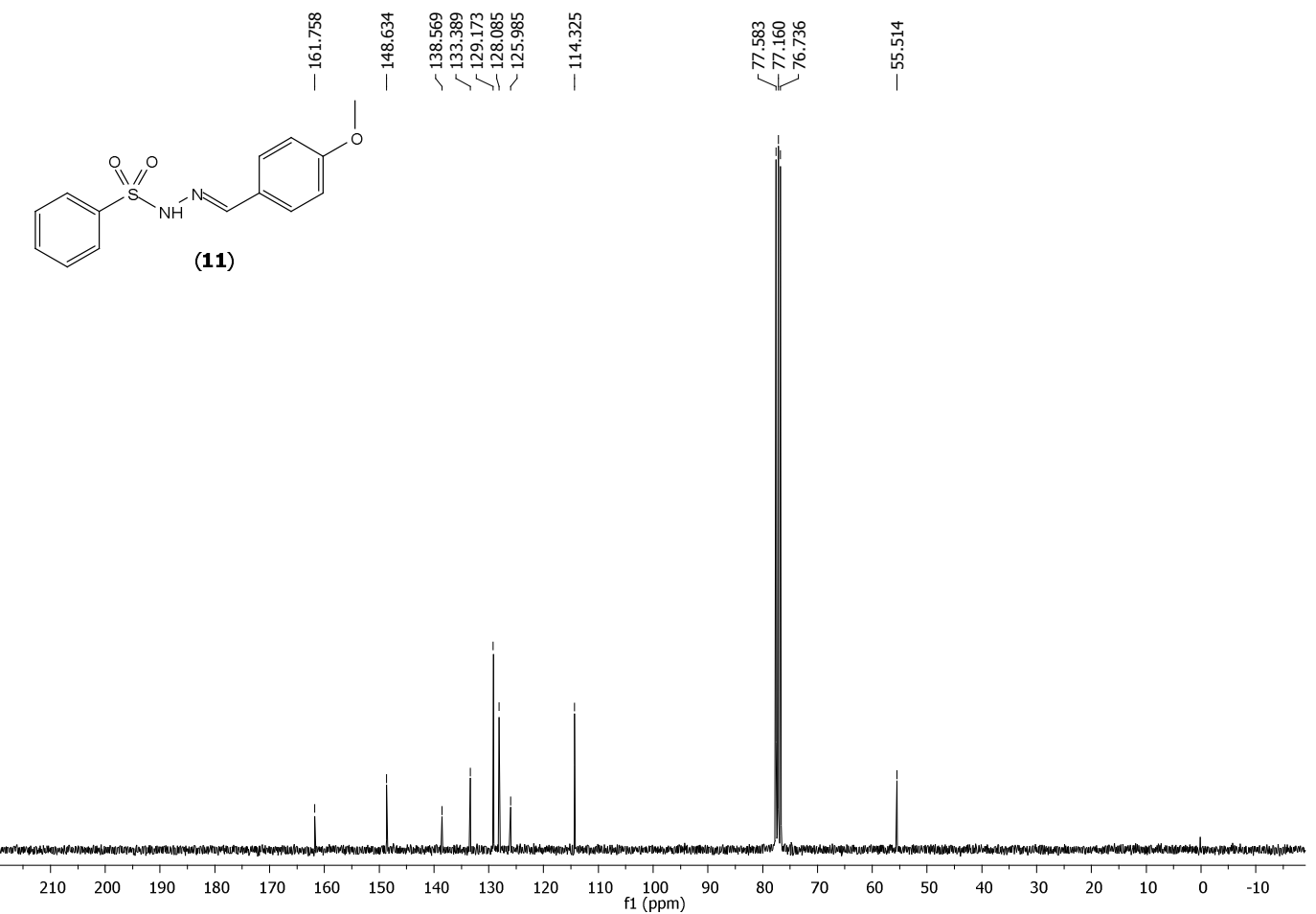

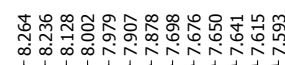

$\backslash 11$
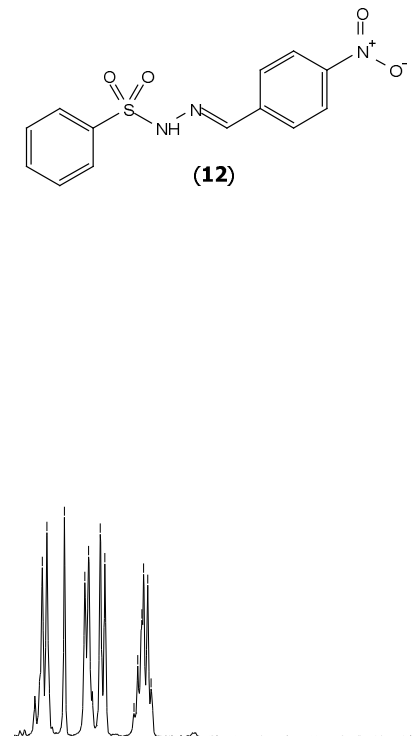

residual sovent

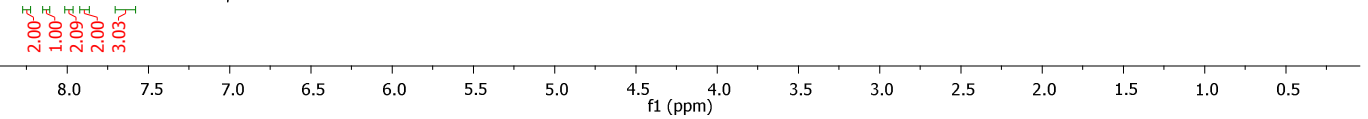




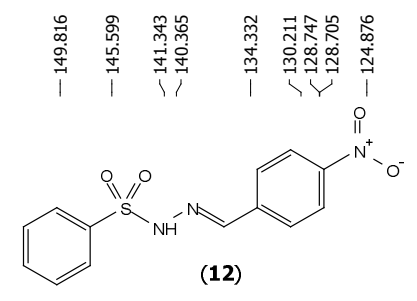

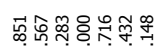

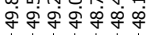
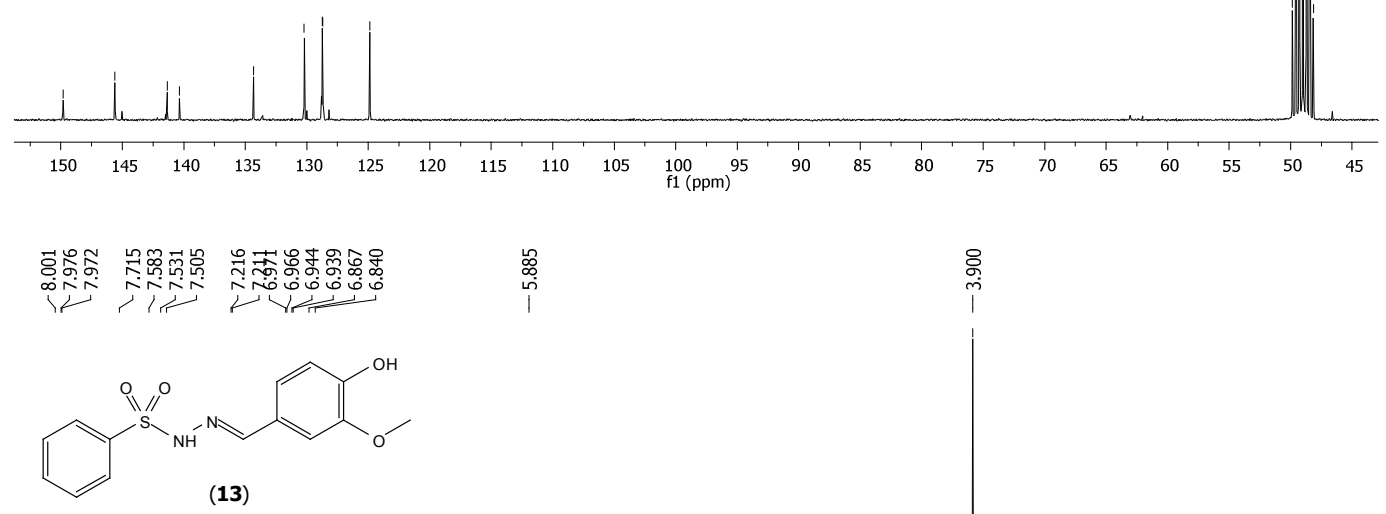

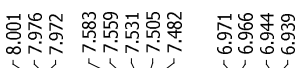
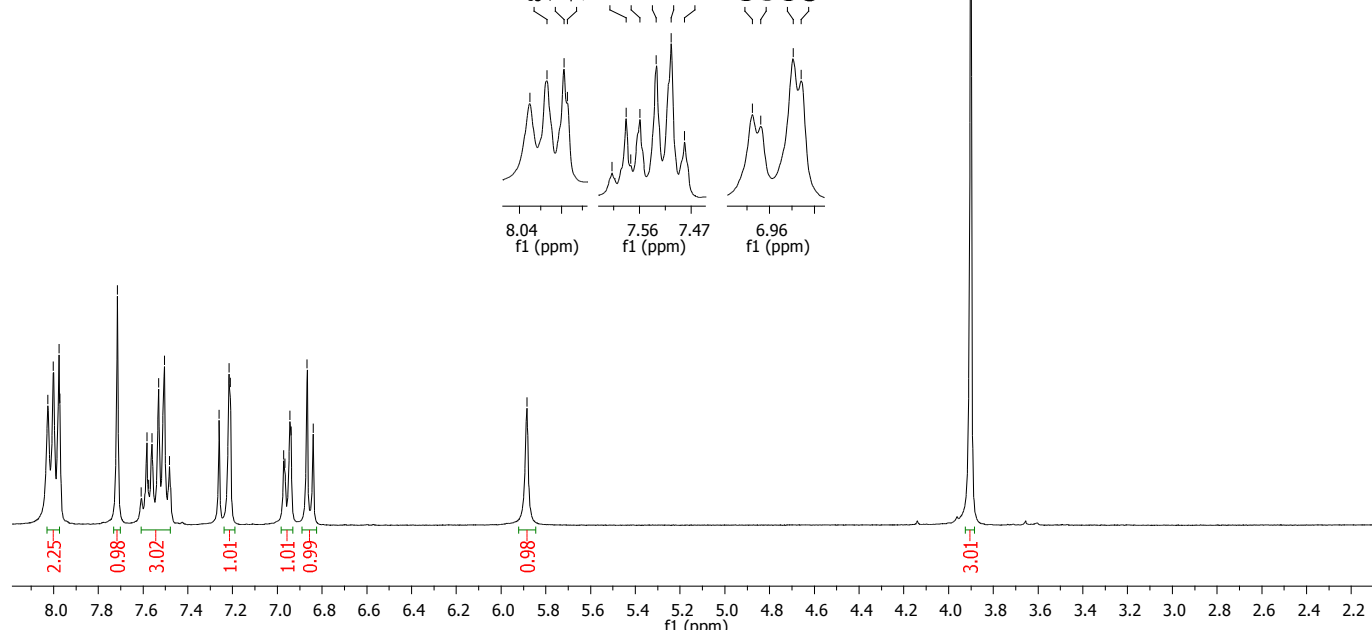


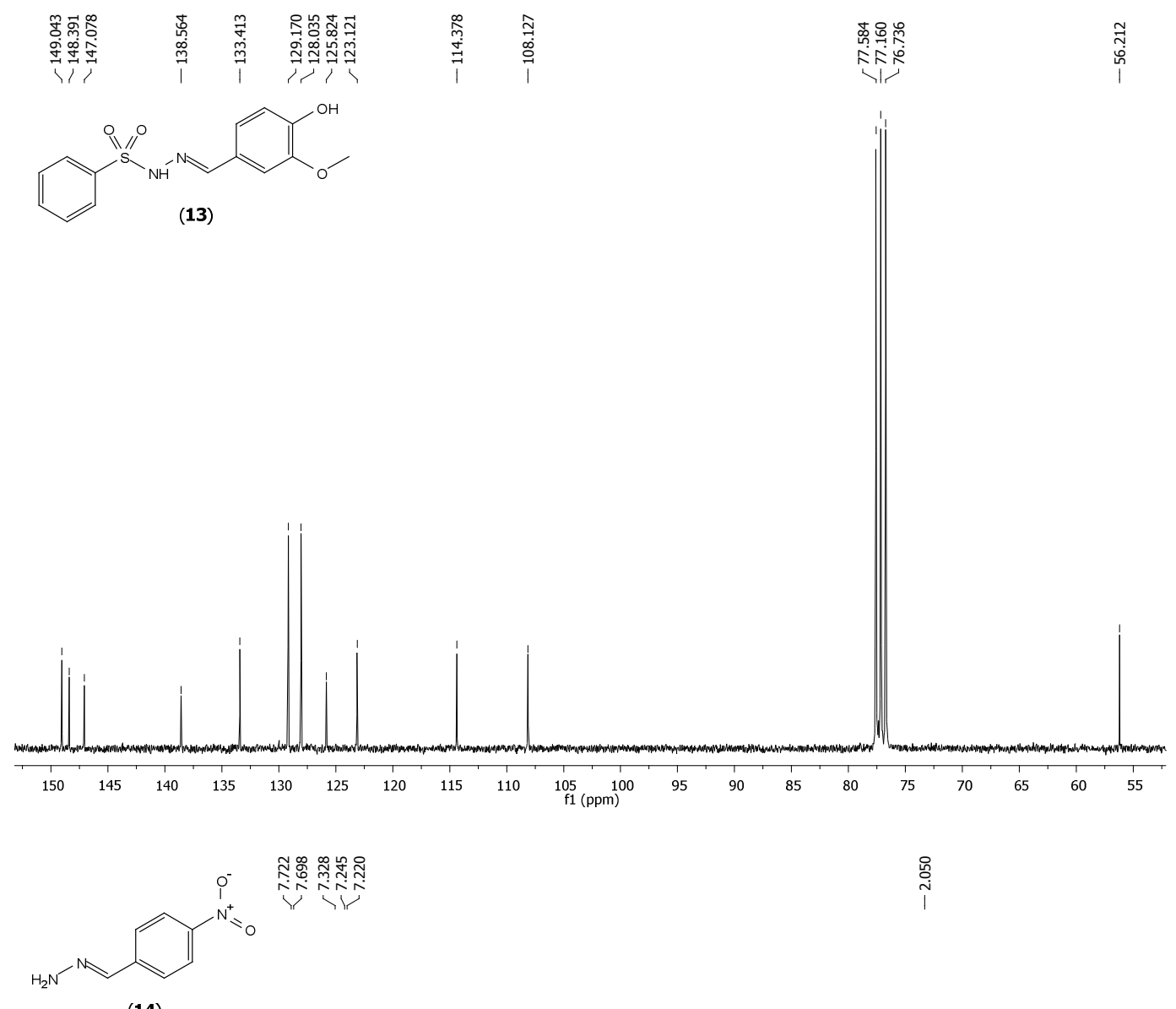

(14)
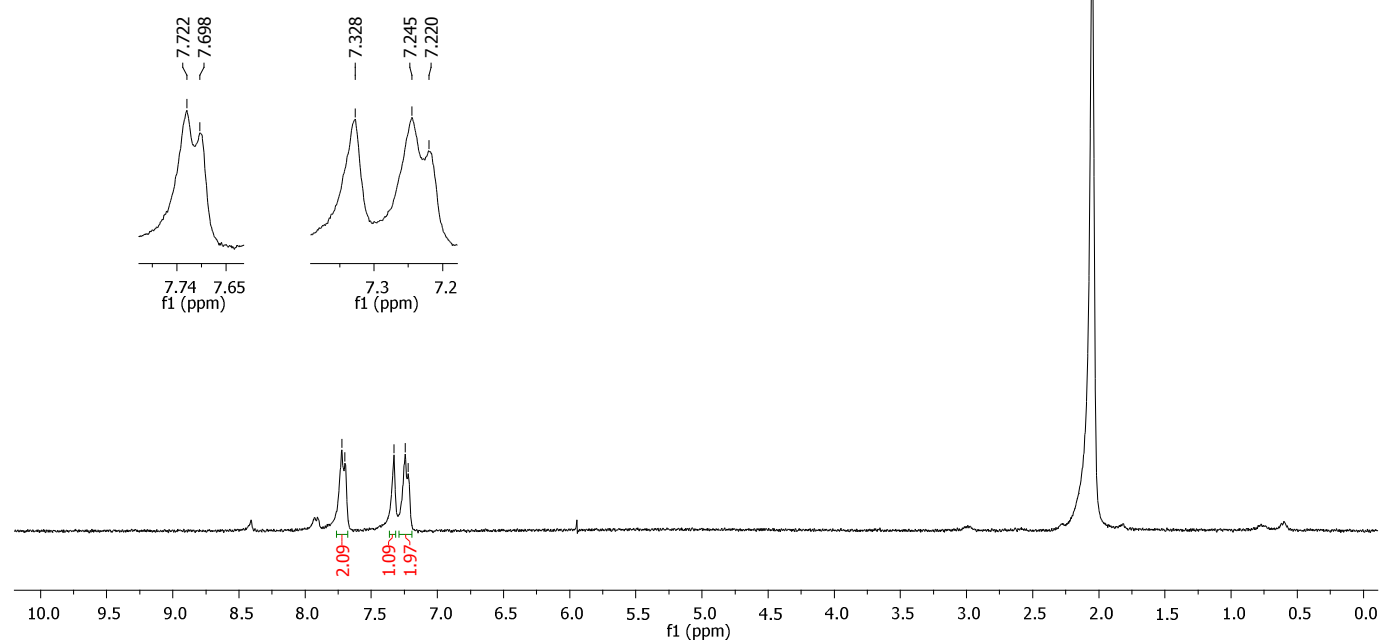


\section{X-ray powder diffraction measurements}

X-ray powder diffraction data were collected at room temperature on a STADI-P powder diffractometer (Stoe ${ }^{\circ}$ Darmstadt, Germany) in transmission geometry by using a $K \alpha_{1}(\lambda=$ $0.7093 \AA$ A) wavelength emitted by a Mo anode and selected by a curved Ge (111) crystal, with a tube voltage of $50 \mathrm{kV}$ and a current of $40 \mathrm{~mA}$. The sample was packed between two acetatecellulose foils, and the sample holder was held spinning during data collection. The diffracted intensities were collected by a silicon microstrip detector, Mythen $1 \mathrm{~K}$ (Dectris', Baden, Switzerland), in the range from $1.6^{\circ}$ to $50.7^{\circ}$, with $200 \mathrm{~s}$ of integration time at each $0.785^{\circ}$.

\subsection{Structure determination procedure}

The crystal structure determination process was conducted on the basis of previous procedures. $^{2-4}$ We used the Topas-Academic v.5 software ${ }^{5}$ to index the powder diffraction pattern. A 3D model of the structure was built using Marvin 15.5.11.0, 2015, ChemAxon (http://www.chemaxon.com), which was then used in the DASH program ${ }^{6}$ to solve the structure. Thus, a Rietveld refinement ${ }^{7,8}$ of the final crystal structure was conducted using the Topas-Academic v.5 software, providing a satisfactory fit as shown below.

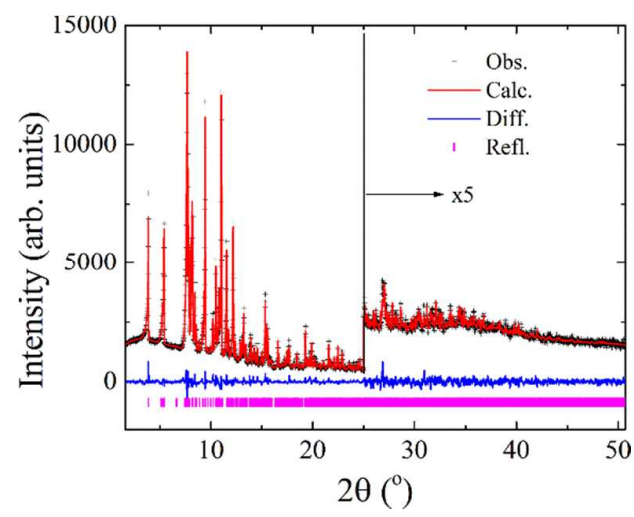

Figure S1. Final Rietveld plot of the $(E)-N^{\prime}-(4-m e t h o x y b e n z y l i d e n e)$ benzenesulfonyl hydrazone. Black crosses represent observed data, the red line indicates the calculated pattern and the blue line at the bottom represent the difference between observed and calculated data. Magenta bars indicate the Bragg peak positions. The pattern was magnified $(5 x)$ after $25^{\circ}$ for better visualization.

In Table S1 are shown details from the Rietveld refinement of the crystal structure. Table S2 shows the final coordinates of the crystal structure. Table \$3 and Table S4 display some selected bond distances and angles, respectively. 
Table S1. Details from the Rietveld refinement of the crystal structure of $(E)-N^{\prime}-(4-$ methoxybenzylidene)benzenesulfonyl hydrazone.

\begin{tabular}{|c|c|}
\hline Chemical formula & $\mathrm{C}_{14} \mathrm{H}_{14} \mathrm{~N}_{2} \mathrm{O}_{3} \mathrm{~S}$ \\
\hline Formula weight $\left(\mathrm{g} \mathrm{mol}^{-1}\right)$ & 290.33 \\
\hline Crystal system & Monoclinic \\
\hline Space group & $P 2_{1} / c \quad$ (Nr. 14) \\
\hline$a, b, c(\AA)$ & $\begin{array}{l}\text { 11.5414(3), 12.2880(6), } \\
10.9161(5)\end{array}$ \\
\hline$B\left({ }^{\circ}\right)$ & $115.252(2)$ \\
\hline Volume $\left(\AA^{3}\right)$ & $1400.19(11)$ \\
\hline$Z, Z^{\prime}$ & 4,1 \\
\hline$\rho_{\text {calc }}\left(\mathrm{g} \mathrm{cm}^{-3}\right)$ & $1.3773(1)$ \\
\hline $\mathrm{T}(\mathrm{K})$ & 298 \\
\hline \multicolumn{2}{|l|}{ Data collection } \\
\hline Diffractometer & STADI P \\
\hline Monochromator & $\mathrm{Ge}(111)$ \\
\hline Wavelength (Å) & 0.7093 \\
\hline $2 \theta$ range $\left({ }^{\circ}\right)$ & $1.6-50.7$ \\
\hline Step size $\left({ }^{\circ}\right)$ & 0.785 \\
\hline Time per step (s) & 200 \\
\hline Refinement & \\
\hline Number of data points & 3276 \\
\hline $\begin{array}{l}\text { Number of contributing } \\
\text { reflections }\end{array}$ & 2643 \\
\hline Number of restraints & 92 \\
\hline Number of refined parameters & 153 \\
\hline$R_{p}(\%)$ & 3.555 \\
\hline$R_{e x p}(\%)$ & 3.200 \\
\hline$R_{w p}(\%)$ & 4.700 \\
\hline$R_{\text {Bragg }}(\%)$ & 1.811 \\
\hline$x^{2}$ & 1.469 \\
\hline
\end{tabular}

Table S2. Final coordinates and equivalent isotropic displacement parameters $\left(U_{\text {iso }}=B_{\text {iso }} / 8 \pi^{2}\right)$ for all atoms in the $(E)-N^{\prime}$-(4-methoxybenzylidene)benzenesulfonyl hydrazone crystal structure.

\begin{tabular}{lllll}
\hline Atom & \multicolumn{1}{c}{$x$} & \multicolumn{1}{c}{$y$} & \multicolumn{1}{c}{$z$} & \multicolumn{1}{c}{$U_{\text {iso }}\left(\AA^{2}\right)$} \\
\hline N1 & $0.262(2)$ & $0.524(2)$ & $0.182(2)$ & 0.03805 \\
C2 & $0.231(2)$ & $0.624(3)$ & $0.176(3)$ & 0.03805 \\
N3 & $0.327(2)$ & $0.483(2)$ & $0.313(2)$ & 0.03805 \\
C4 & $0.148(3)$ & $0.679(2)$ & $0.047(3)$ & 0.03805 \\
H5 & $0.288(19)$ & $0.680(16)$ & $0.260(19)$ & 0.04567 \\
S6 & $0.4206(9)$ & $0.3800(7)$ & $0.3274(9)$ & 0.03805 \\
H7 & $0.37(2)$ & $0.537(17)$ & $0.389(19)$ & 0.04567 \\
C8 & $0.069(3)$ & $0.619(3)$ & $-0.065(3)$ & 0.03805 \\
C9 & $0.151(3)$ & $0.792(3)$ & $0.036(3)$ & 0.03805 \\
C10 & $0.521(3)$ & $0.416(2)$ & $0.252(3)$ & 0.03805 \\
O11 & $0.4953(16)$ & $0.3667(14)$ & $0.4711(16)$ & 0.03805 \\
O12 & $0.3392(16)$ & $0.2930(14)$ & $0.2479(17)$ & 0.03805 \\
C13 & $-0.005(3)$ & $0.672(2)$ & $-0.185(3)$ & 0.03805 \\
H14 & $0.064(17)$ & $0.532(15)$ & $-0.060(19)$ & 0.04567 \\
C15 & $0.078(3)$ & $0.840(2)$ & $-0.084(3)$ & 0.03805 \\
H16 & $0.211(17)$ & $0.843(15)$ & $0.12(2)$ & 0.04567 \\
C17 & $0.598(3)$ & $0.504(3)$ & $0.298(3)$ & 0.03805 \\
C18 & $0.517(3)$ & $0.356(2)$ & $0.142(3)$ & 0.03805 \\
C19 & $0.001(3)$ & $0.781(3)$ & $-0.194(3)$ & 0.03805
\end{tabular}




\begin{tabular}{lllll} 
H20 & $-0.066(17)$ & $0.623(16)$ & $-0.271(19)$ & 0.04567 \\
H21 & $0.080(17)$ & $0.928(17)$ & $-0.093(19)$ & 0.04567 \\
C22 & $0.676(3)$ & $0.532(3)$ & $0.236(3)$ & 0.03805 \\
H23 & $0.600(19)$ & $0.552(15)$ & $0.38(2)$ & 0.04567 \\
C24 & $0.596(3)$ & $0.386(3)$ & $0.083(3)$ & 0.03805 \\
H25 & $0.46(2)$ & $0.285(16)$ & $0.10(2)$ & 0.04567 \\
O26 & $-0.0661(17)$ & $0.8399(14)$ & $-0.3099(17)$ & 0.03805 \\
C27 & $0.675(3)$ & $0.473(3)$ & $0.130(3)$ & 0.03805 \\
H28 & $0.739(17)$ & $0.603(16)$ & $0.272(18)$ & 0.04567 \\
H29 & $0.595(16)$ & $0.339(16)$ & $-0.003(16)$ & 0.04567 \\
C30 & $-0.152(3)$ & $0.781(3)$ & $-0.428(3)$ & 0.03805 \\
H31 & $0.736(17)$ & $0.496(16)$ & $0.081(19)$ & 0.04567 \\
H32 & $-0.218(18)$ & $0.730(18)$ & $-0.404(19)$ & 0.04567 \\
H33 & $-0.211(17)$ & $0.839(17)$ & $-0.505(18)$ & 0.04567 \\
H34 & $-0.100(18)$ & $0.731(17)$ & $-0.470(19)$ & 0.04567 \\
\hline
\end{tabular}

Table S3. Some selected bond distances of (E)-N'-(4-methoxybenzylidene)benzenesulfonyl hydrazone.

\begin{tabular}{ll}
\hline Bonds & Lengths (Å) \\
\hline N1-C2 & $1.27(4)$ \\
N1-N3 & $1.40(3)$ \\
C2-C4 & $1.49(4)$ \\
N3-S6 & $1.63(3)$ \\
C4-C8 & $1.39(4)$ \\
C4-C9 & $1.40(4)$ \\
S6-C10 & $1.74(4)$ \\
S6-O11 & $1.44(2)$ \\
S6-O12 & $1.44(2)$ \\
C8-C13 & $1.39(4)$ \\
C9-C15 & $1.35(4)$ \\
C10-C17 & $1.35(4)$ \\
C10-C18 & $1.39(5)$ \\
C13-C19 & $1.35(4)$ \\
C15-C19 & $1.36(4)$ \\
C17-C22 & $1.38(6)$ \\
C18-C24 & $1.37(6)$ \\
C19-O26 & $1.37(3)$ \\
C22-C27 & $1.37(5)$ \\
C24-C27 & $1.36(5)$ \\
O26-C30 & $1.44(3)$ \\
\hline
\end{tabular}

Table S4. Some selected bond angles of $(E)-N^{\prime}$-(4-methoxybenzylidene)benzenesulfonyl hydrazone.

\begin{tabular}{ll}
\hline Bonds & Angles $\left(^{\circ}\right)$ \\
\hline C2-N1-N3 & $115(2)$ \\
N1-C2-C4 & $123(3)$ \\
N1-N3-S6 & $115(2)$ \\
C2-C4-C8 & $121(3)$ \\
C2-C4-C9 & $120(3)$ \\
C8-C4-C9 & $119(3)$ \\
N3-S6-C10 & $108(1)$ \\
N3-S6-O11 & $105(1)$ \\
N3-S6-O12 & $106(1)$ \\
C10-S6-O11 & $110(1)$ \\
C10-S6-O12 & $107(1)$ \\
O11-S6-O12 & $120(1)$
\end{tabular}




\begin{tabular}{ll}
$\mathrm{C} 4-\mathrm{C} 8-\mathrm{C} 13$ & $120(3)$ \\
$\mathrm{C} 4-\mathrm{C} 9-\mathrm{C} 15$ & $119(3)$ \\
$\mathrm{S} 6-\mathrm{C} 10-\mathrm{C} 17$ & $119(3)$ \\
$\mathrm{S} 6-\mathrm{C} 10-\mathrm{C} 18$ & $119(2)$ \\
$\mathrm{C} 17-\mathrm{C} 10-\mathrm{C} 18$ & $121(3)$ \\
$\mathrm{C} 8-\mathrm{C} 13-\mathrm{C} 19$ & $120(3)$ \\
$\mathrm{C} 9-\mathrm{C} 15-\mathrm{C} 19$ & $122(3)$ \\
$\mathrm{C} 10-\mathrm{C} 17-\mathrm{C} 22$ & $119(3)$ \\
$\mathrm{C} 10-\mathrm{C} 18-\mathrm{C} 24$ & $119(3)$ \\
$\mathrm{C} 13-\mathrm{C} 19-\mathrm{C} 15$ & $120(3)$ \\
$\mathrm{C} 13-\mathrm{C} 19-\mathrm{O} 26$ & $124(3)$ \\
$\mathrm{C} 15-\mathrm{C} 19-\mathrm{O} 26$ & $116(3)$ \\
$\mathrm{C} 17-\mathrm{C} 22-\mathrm{C} 27$ & $120(3)$ \\
$\mathrm{C} 18-\mathrm{C} 24-\mathrm{C} 27$ & $121(3)$ \\
$\mathrm{C} 19-\mathrm{O} 26-\mathrm{C} 30$ & $117(2)$ \\
$\mathrm{C} 22-\mathrm{C} 27-\mathrm{C} 24$ & $120(3)$ \\
\hline
\end{tabular}

Figure S2 and Figure S3 reveal atoms labelling and intermolecular interactions of $(E)-N^{\prime}-(4-$ methoxybenzylidene)benzenesulfonyl hydrazone, respectively.

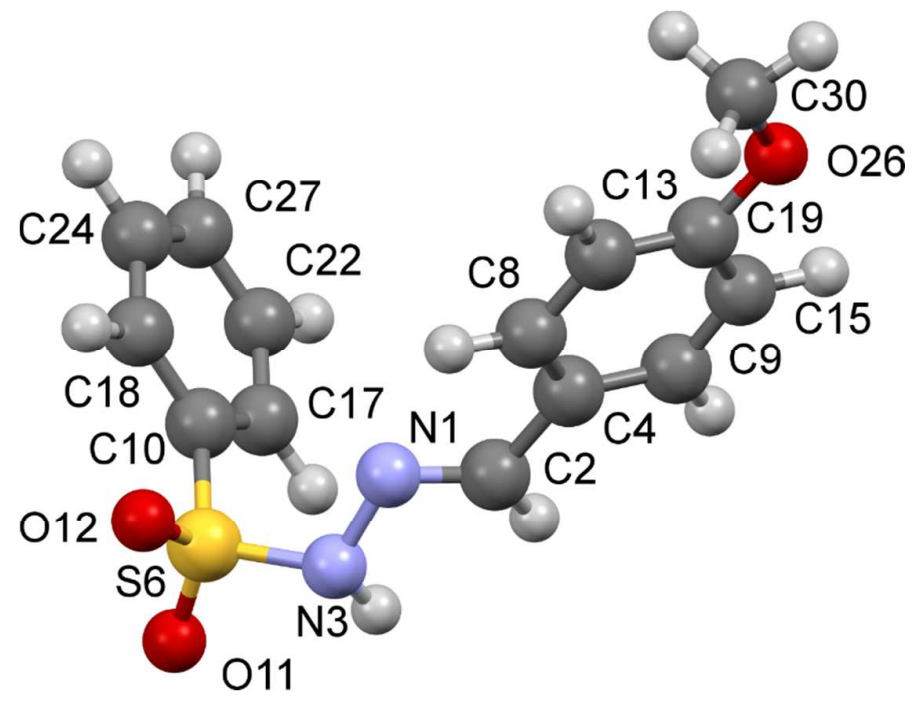

Figure S1. View of the molecule of $(E)-N^{\prime}$-(4-methoxybenzylidene)benzenesulfonyl hydrazone showing the non-hydrogen atoms labelling. 


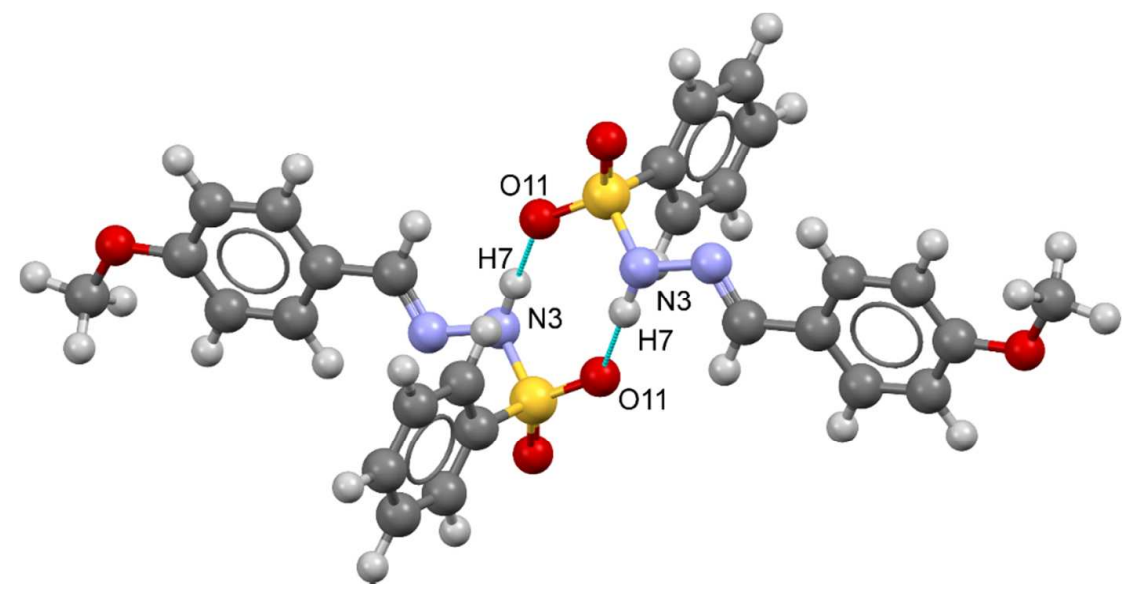

Figure S2. Intermolecular hydrogen bond interaction (cyan lines) of $(E)-N^{\prime}-(4-$ methoxybenzylidene)benzenesulfonyl hydrazone. 
5. HRMS of compounds 1 to 13

N'-benzylidenebutane-1-sulfonyl hydrazone (1)
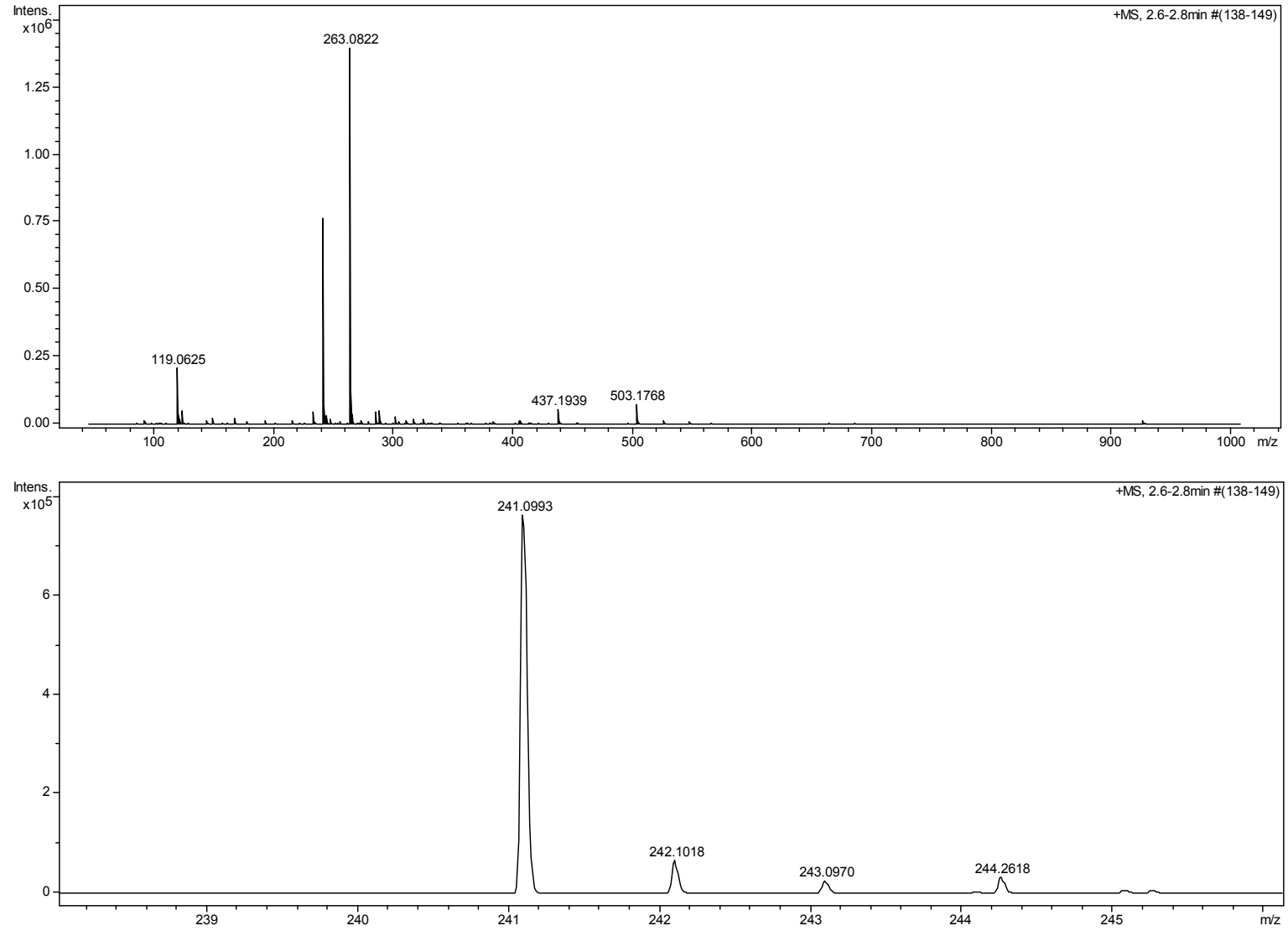

N'-(4-methoxybenzylidene) butane-1- sulfonyl hydrazone (2)

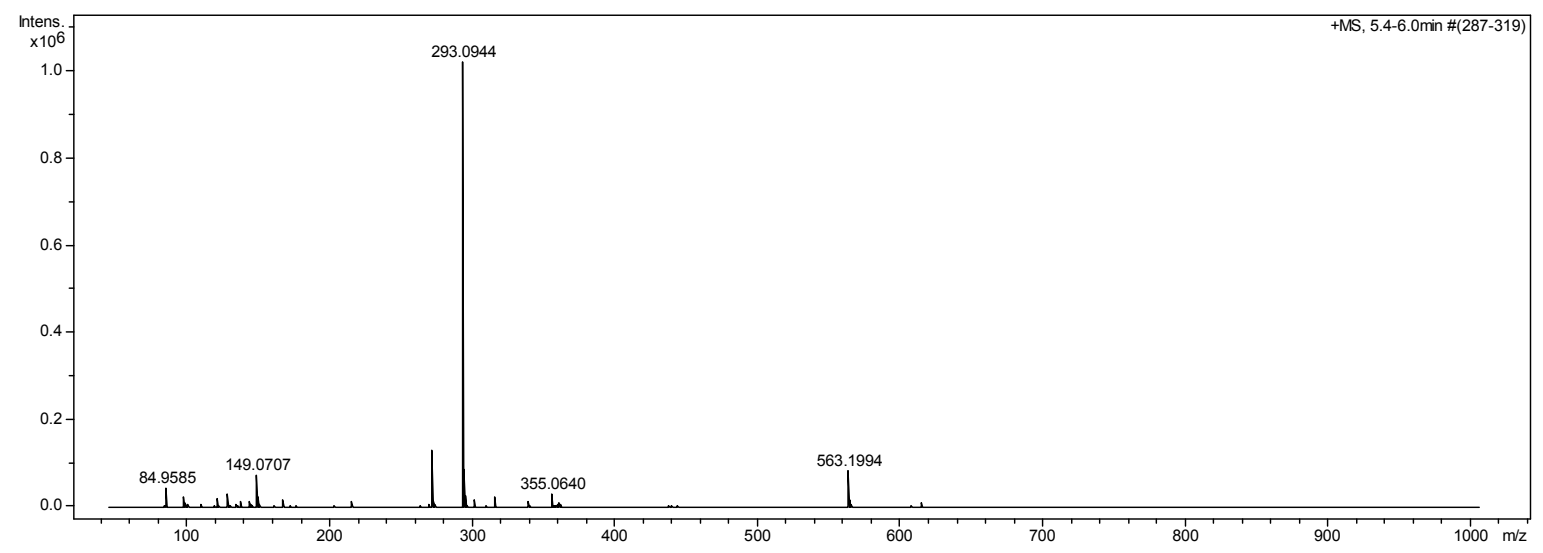




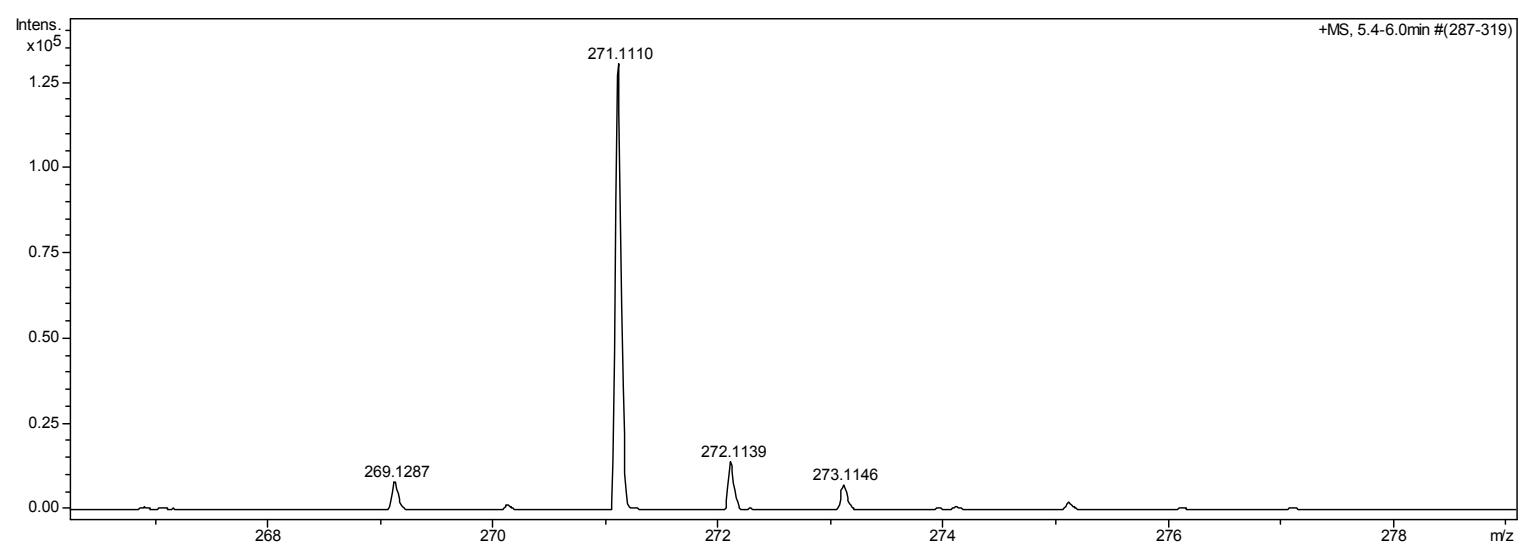

$N^{\prime}$-(4-nitrobenzylidene) butane-1- sulfonyl hydrazone (3)
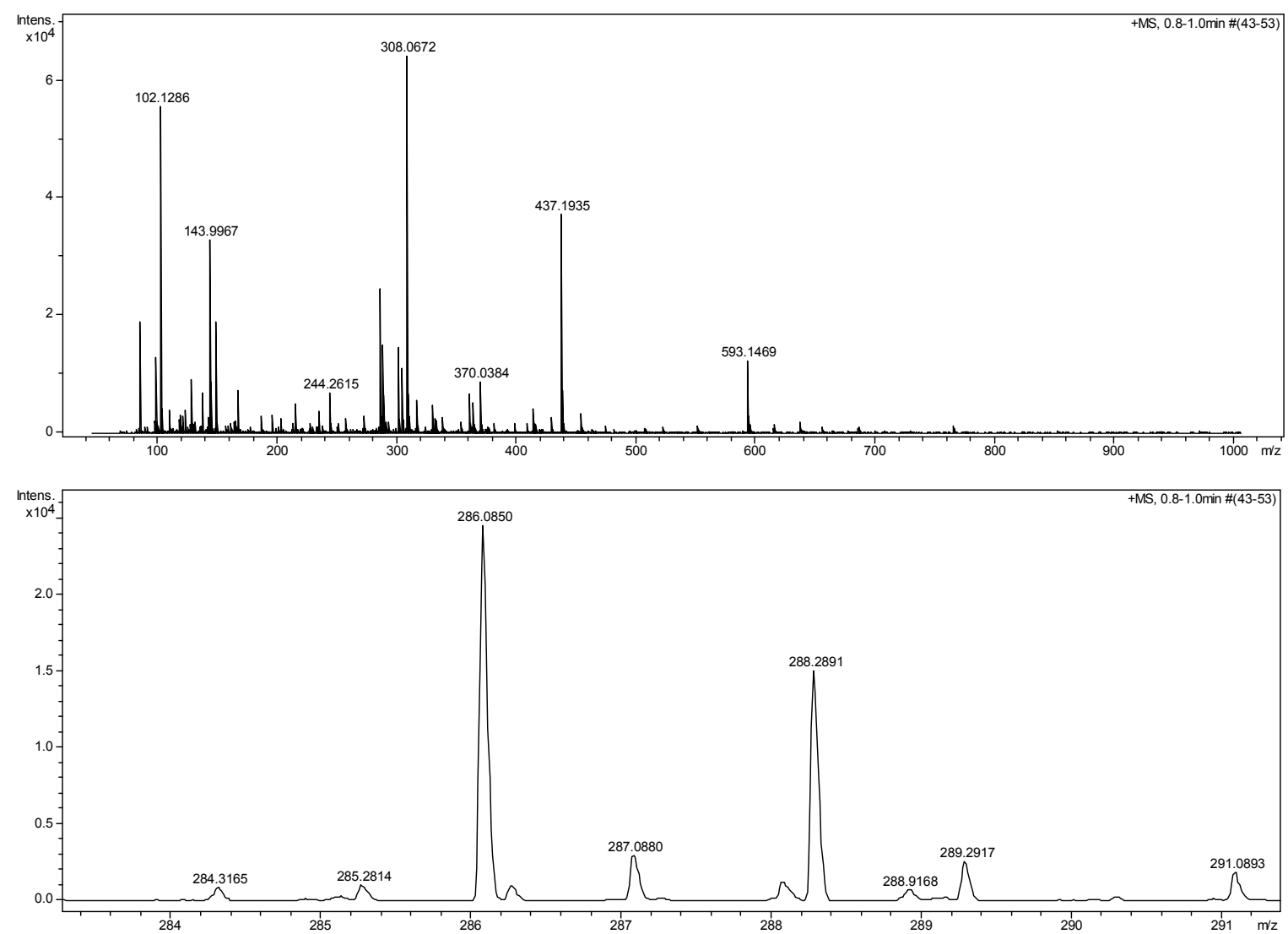
N'-(4-hydroxy-3-methoxybenzylidene) butane-1- sulfonyl hydrazone (4)
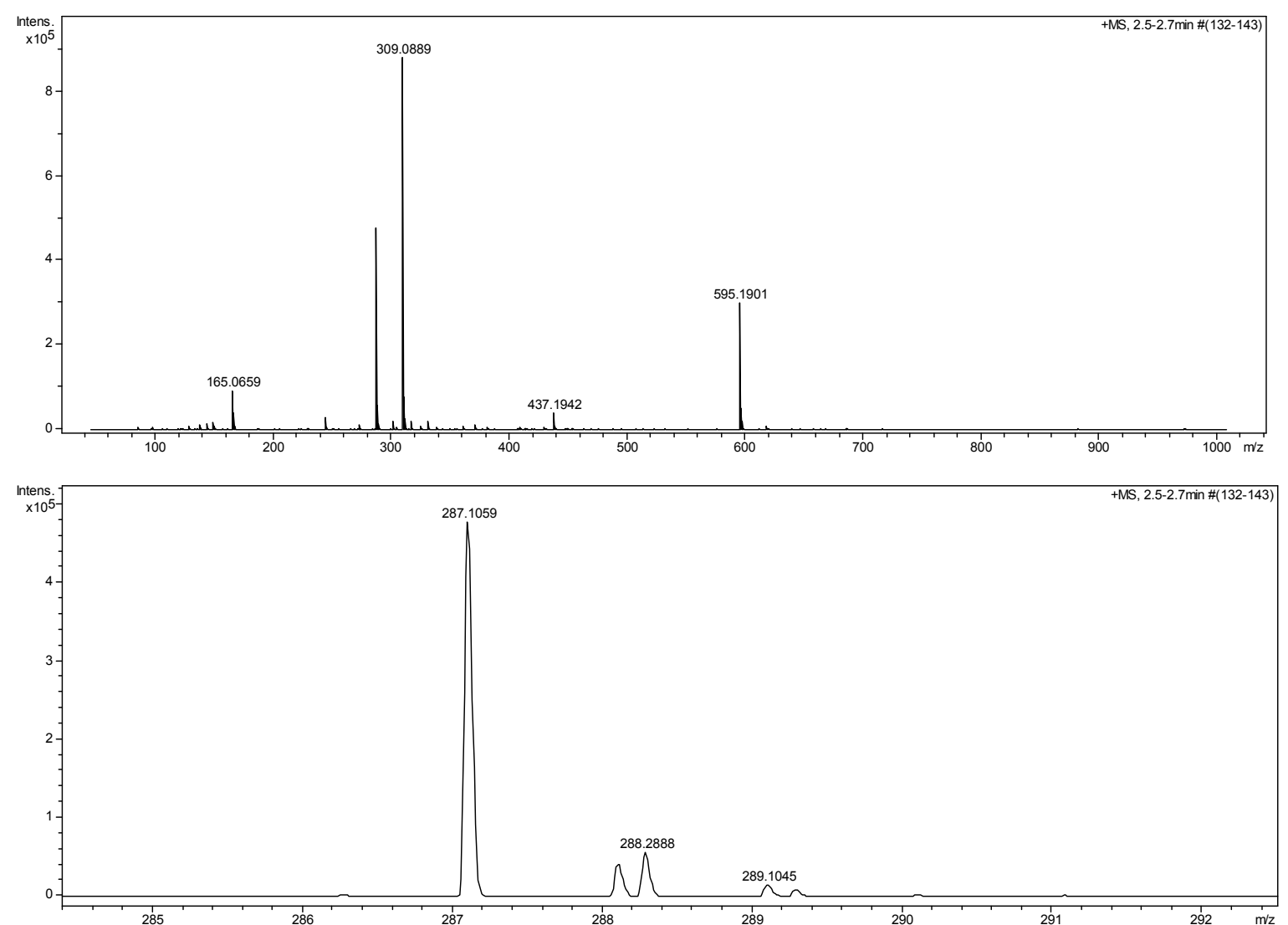

N'-(thiophen-2-ylmethylene) butane-1- sulfonyl hydrazone (5)

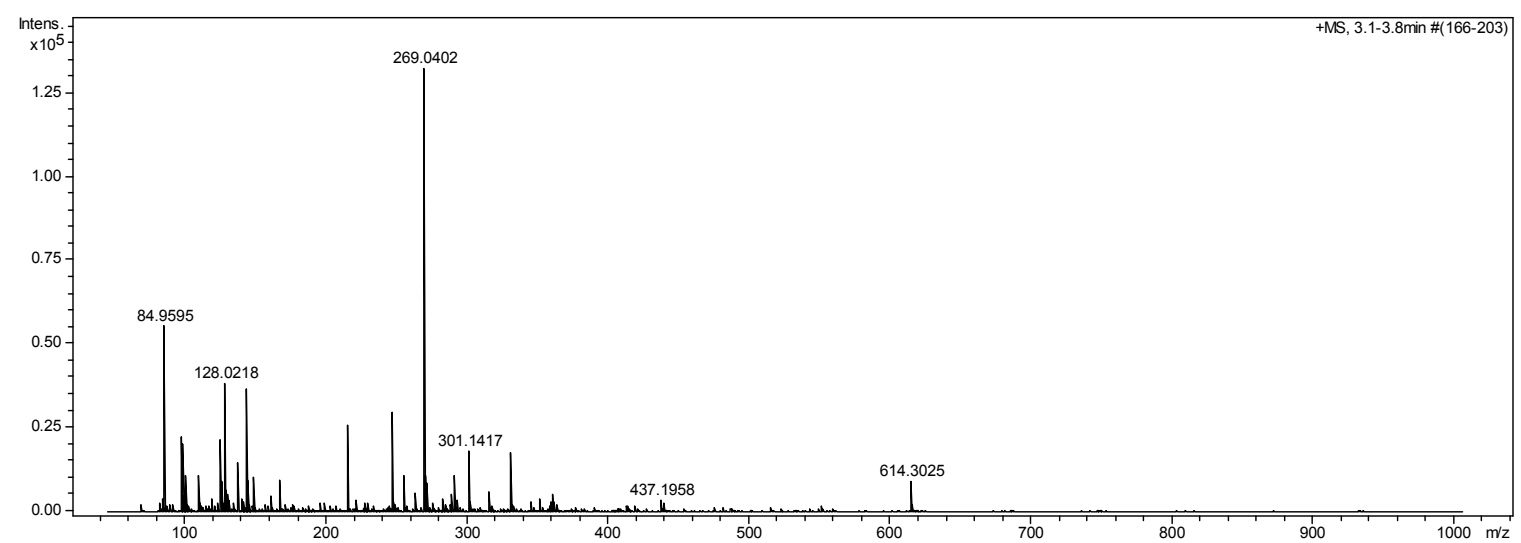




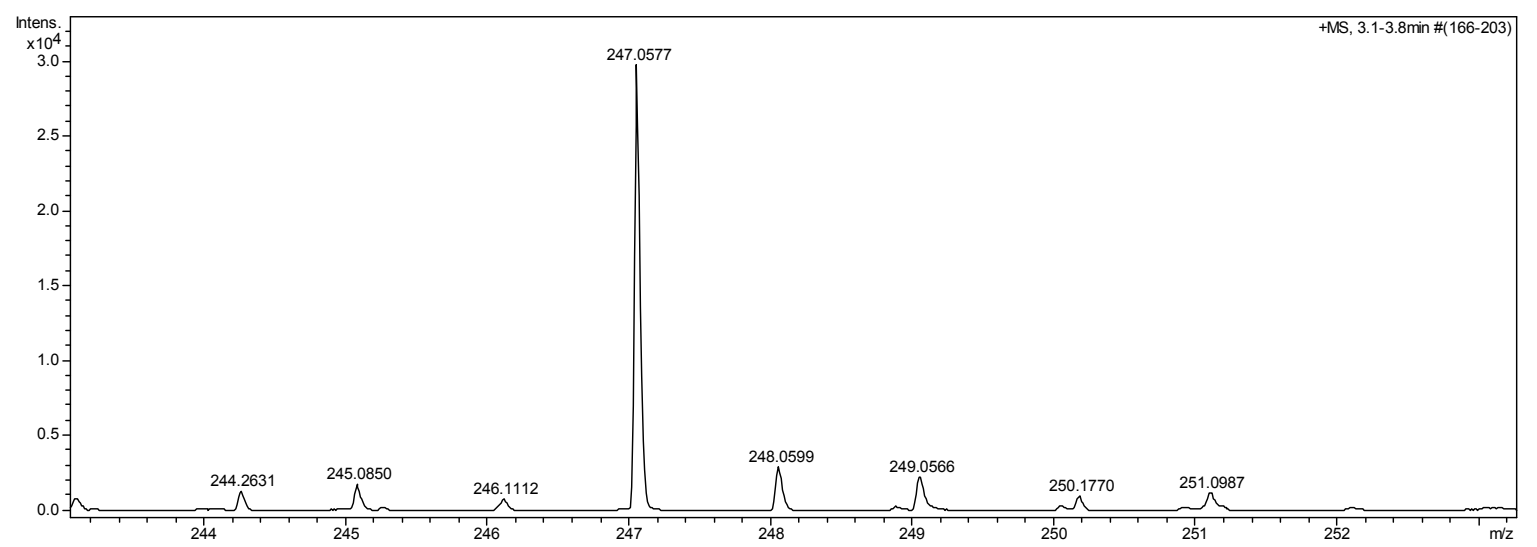

$N^{\prime}$-benzylidene-ethane-sulfonyl hydrazone (6)
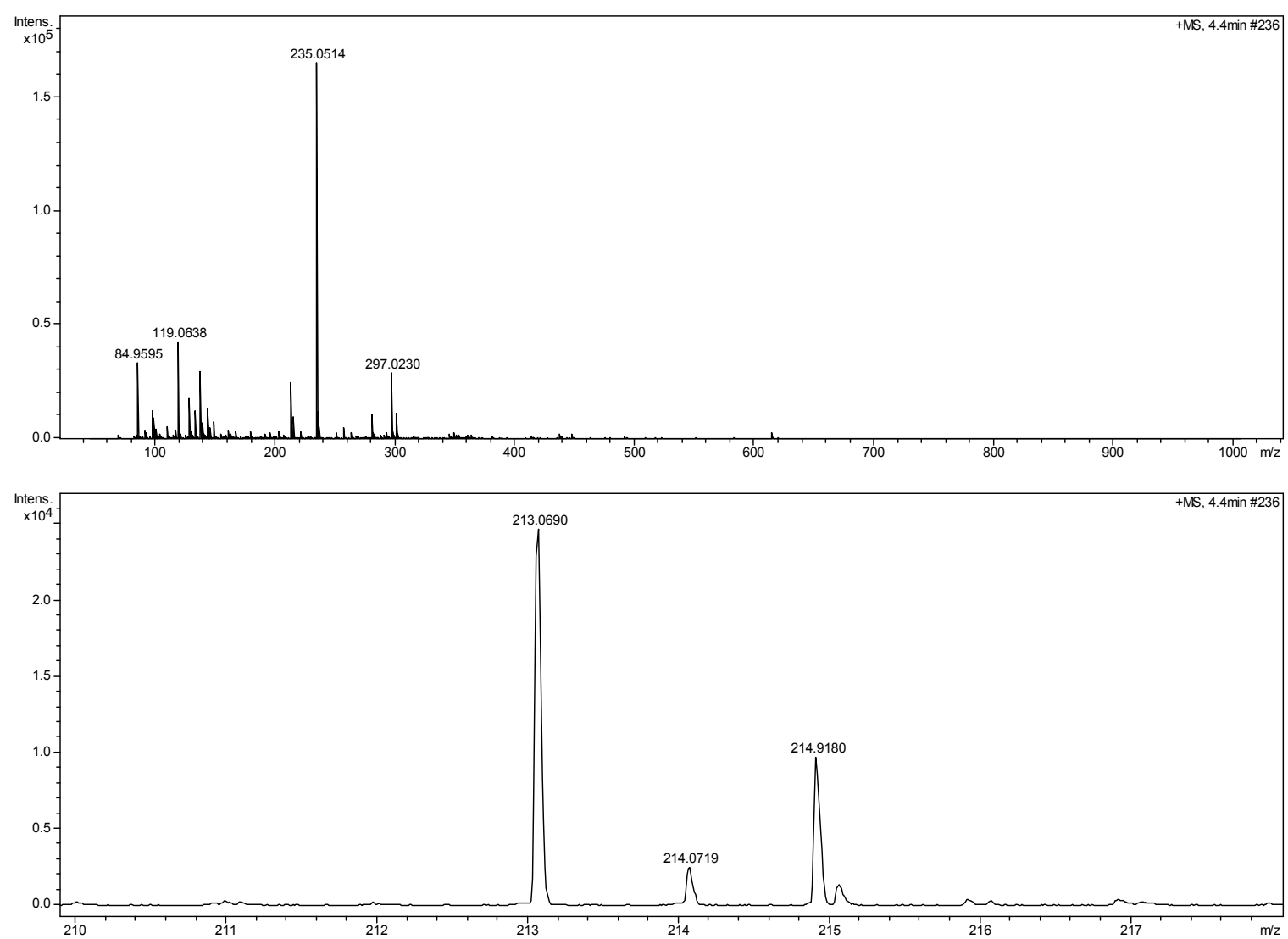
N'-(4-methoxybenzylidene) ethane-sulfonyl hydrazone (7)
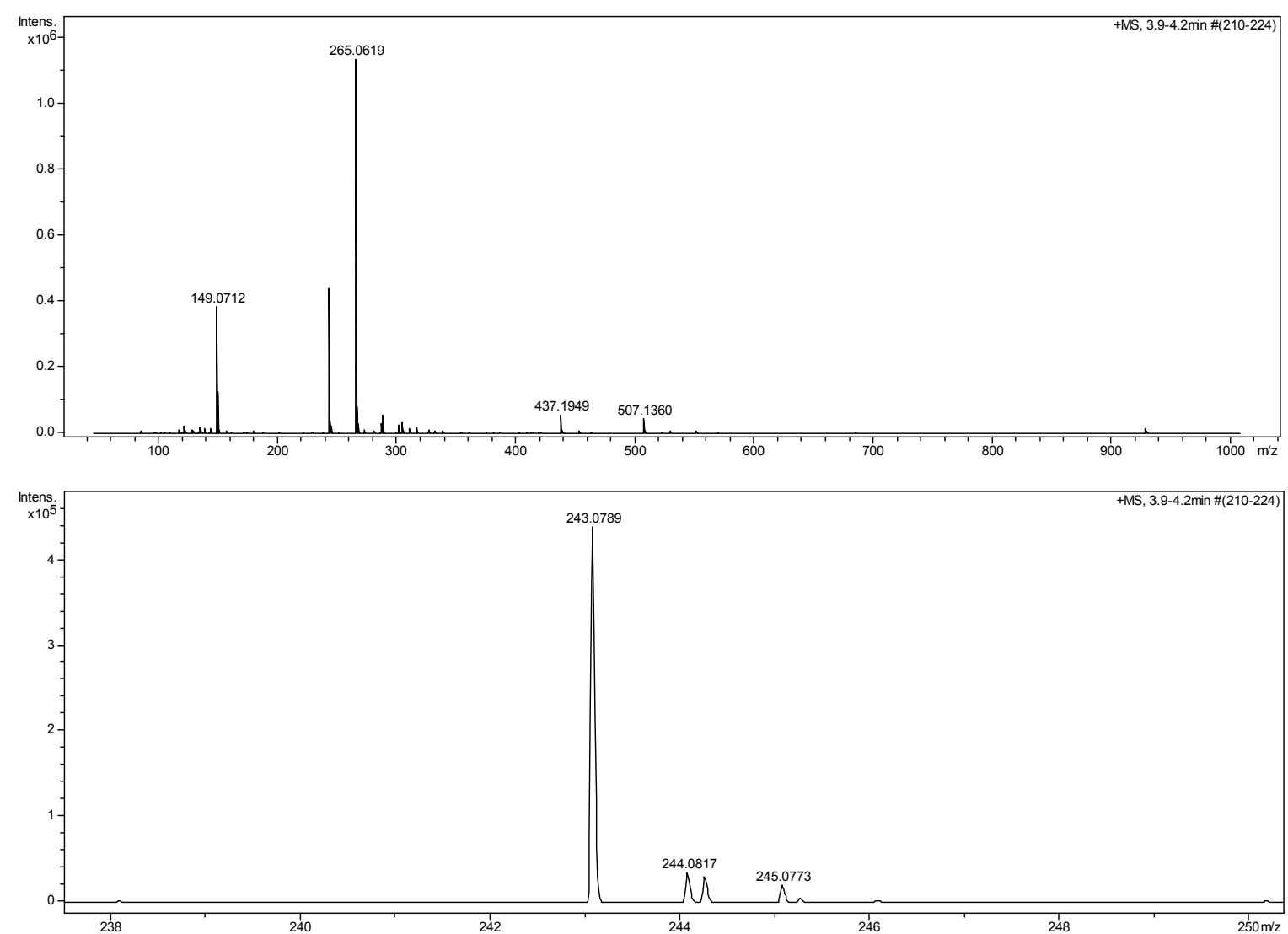

N'-(4-nitrobenzylidene) ethane-sulfonyl hydrazone (8)

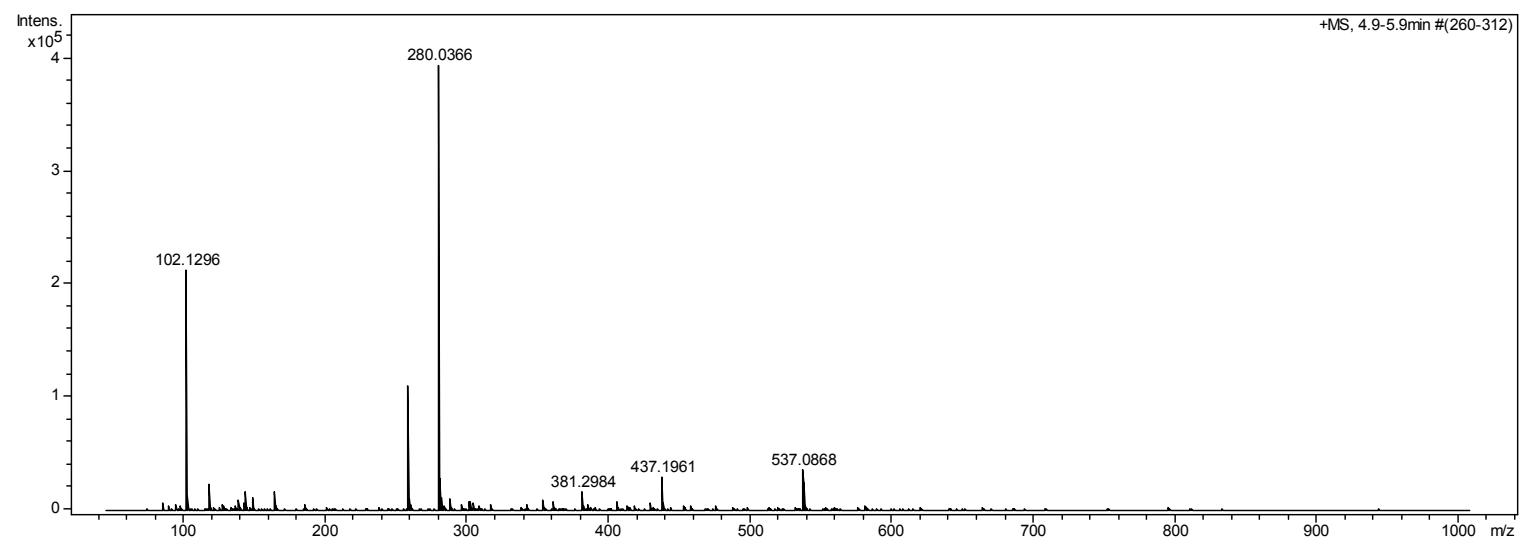




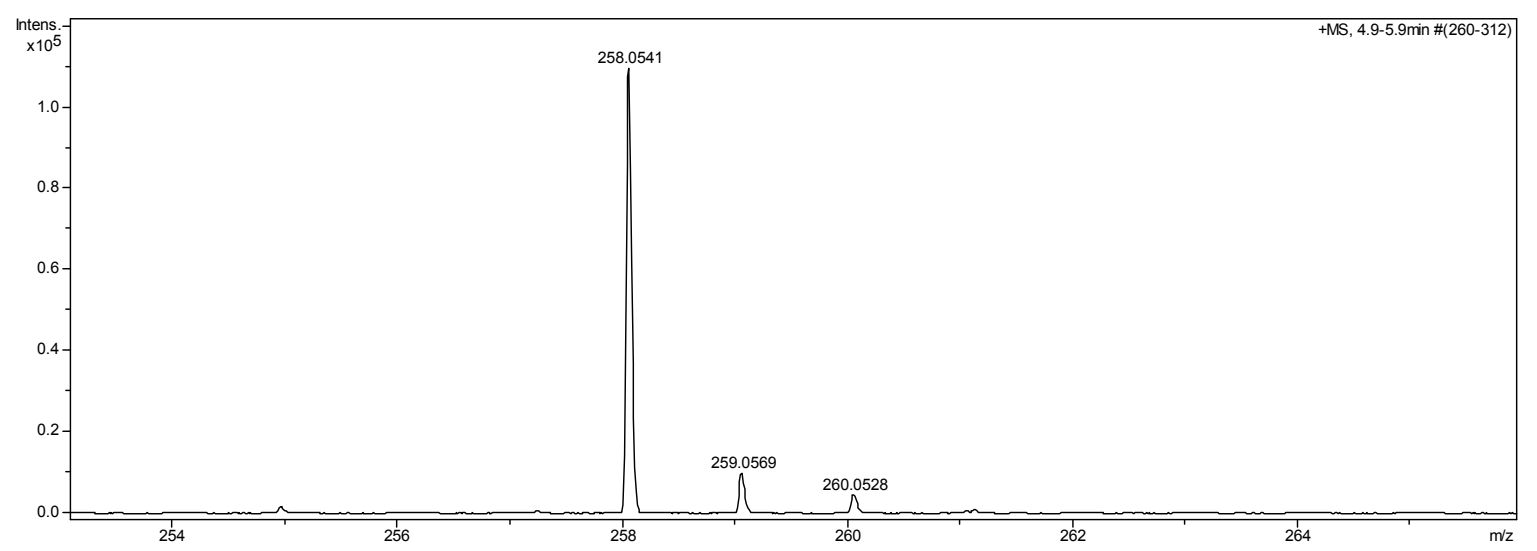

N'-(4-hydroxy-3-methoxybenzylidene) ethanesulfonyl hydrazone (9)
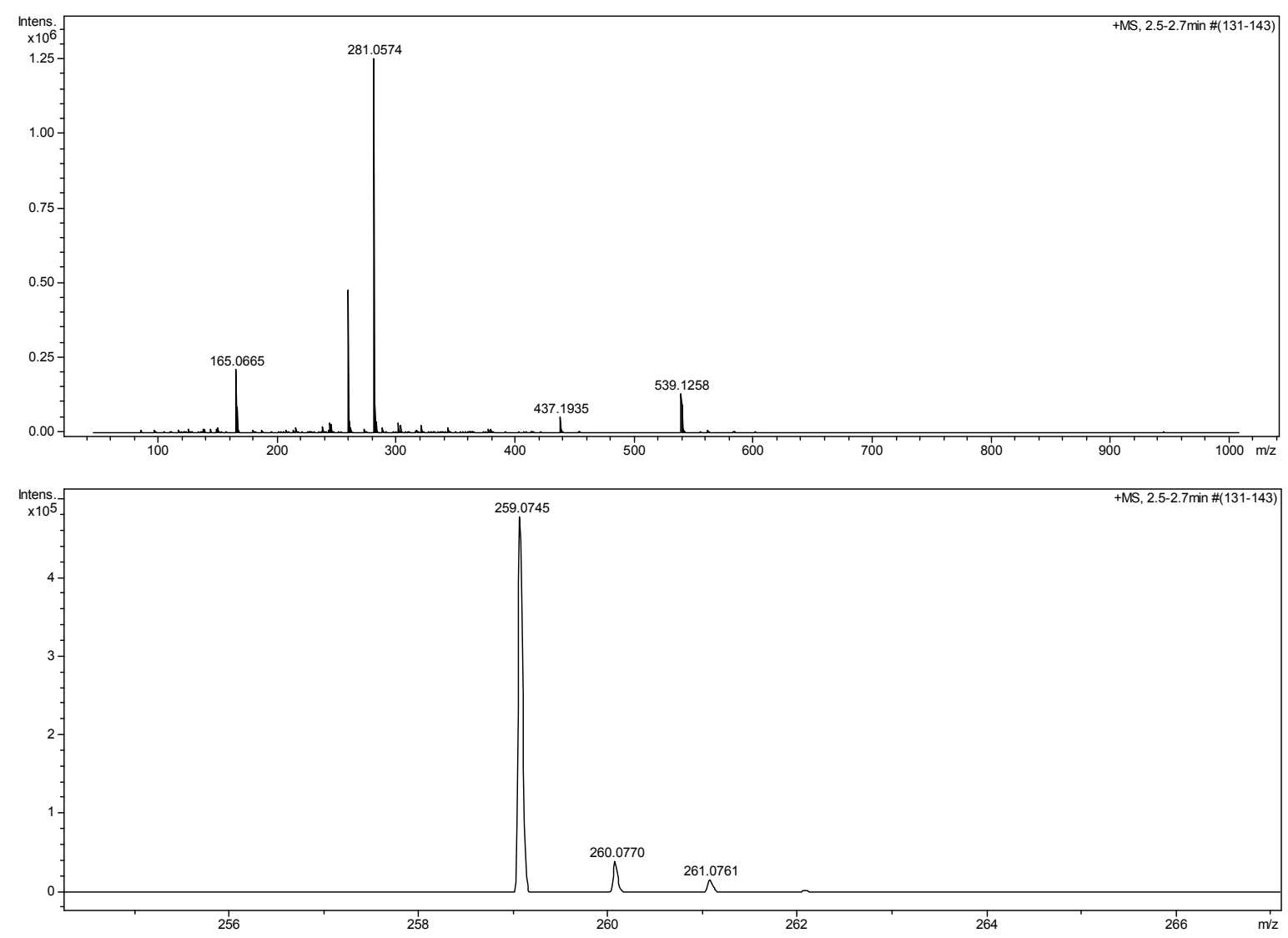
$N^{\prime}$-benzylidenebenzenesulfonyl hydrazone (10)
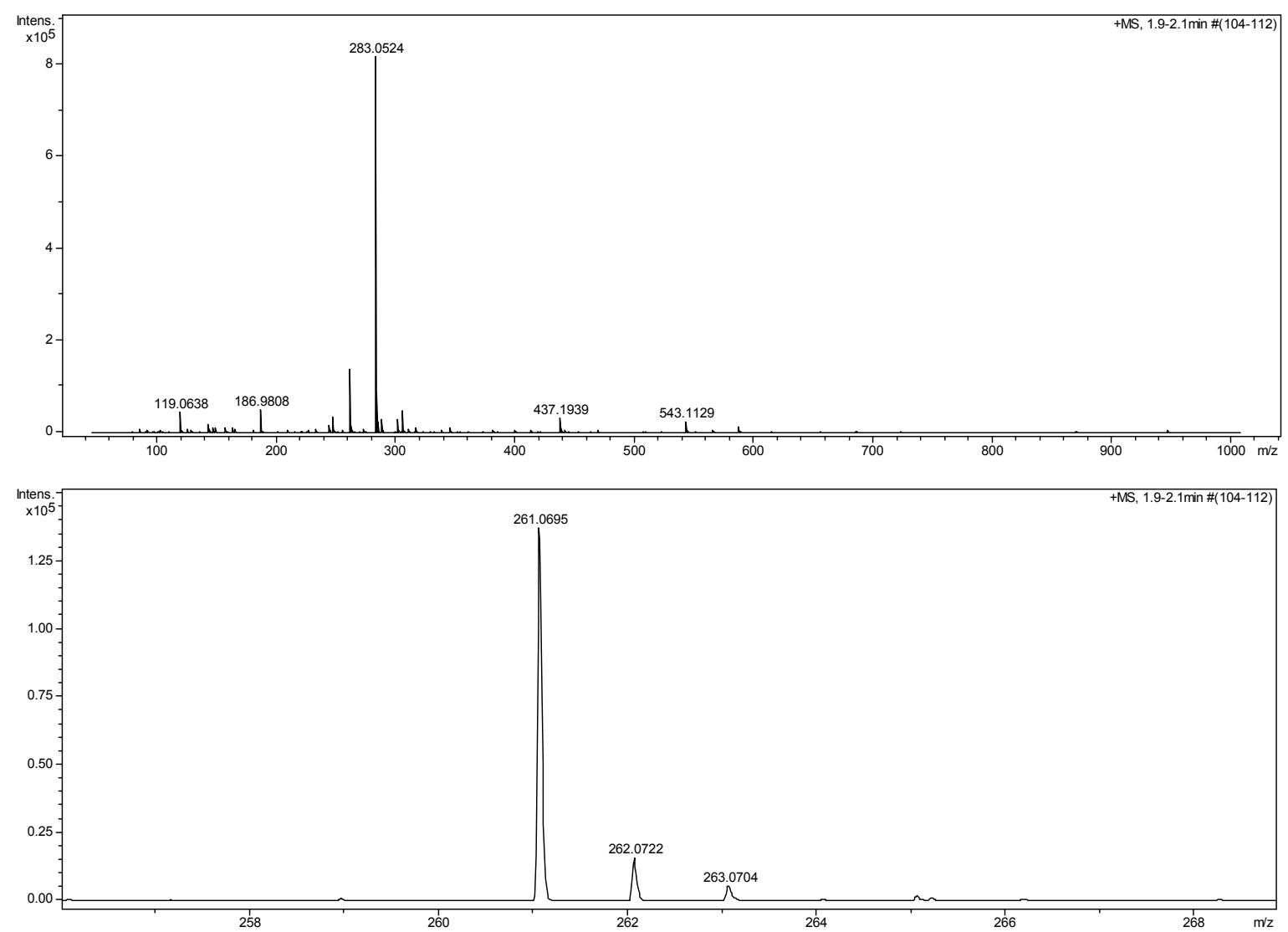

N'-(4-methoxybenzylidene) benzenesulfonyl hydrazone (11)

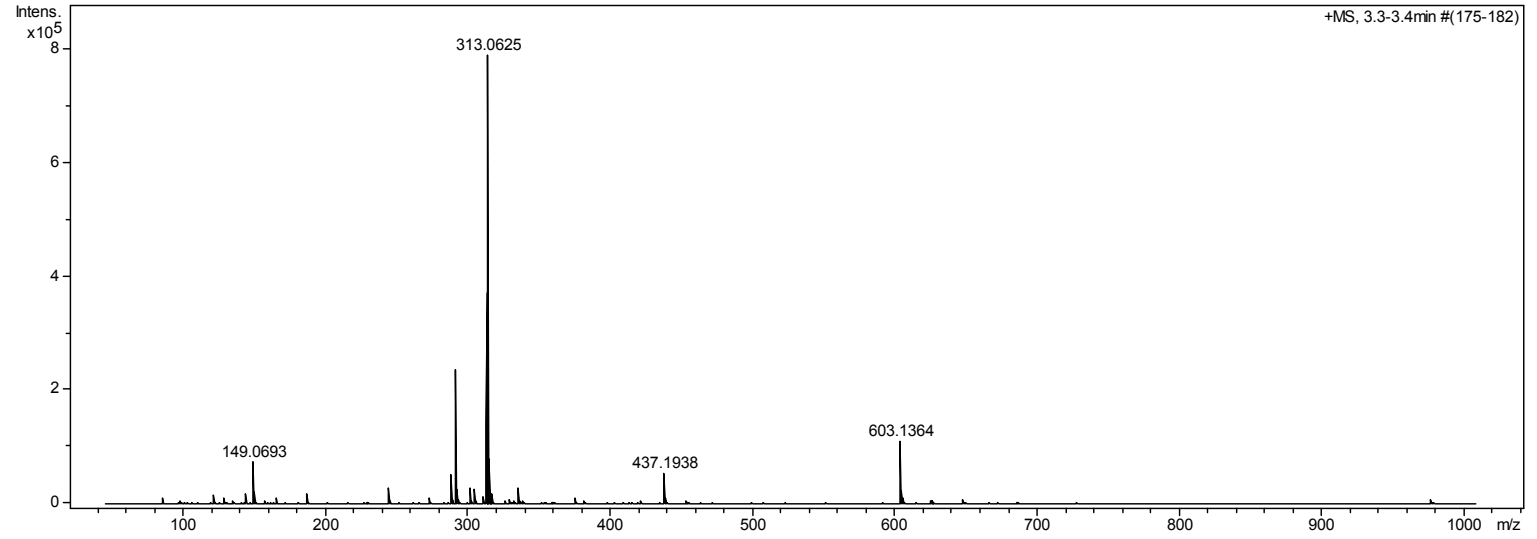




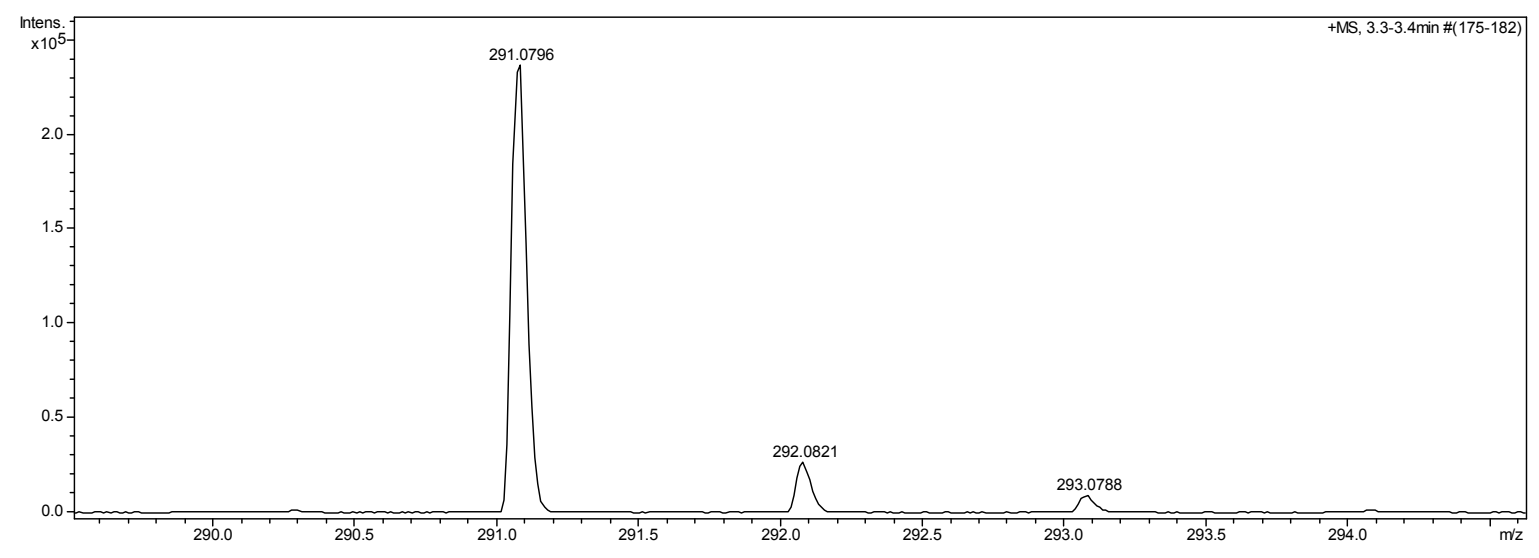

N'-(4-nitrobenzylidene) benzenesulfonyl hydrazone (12)
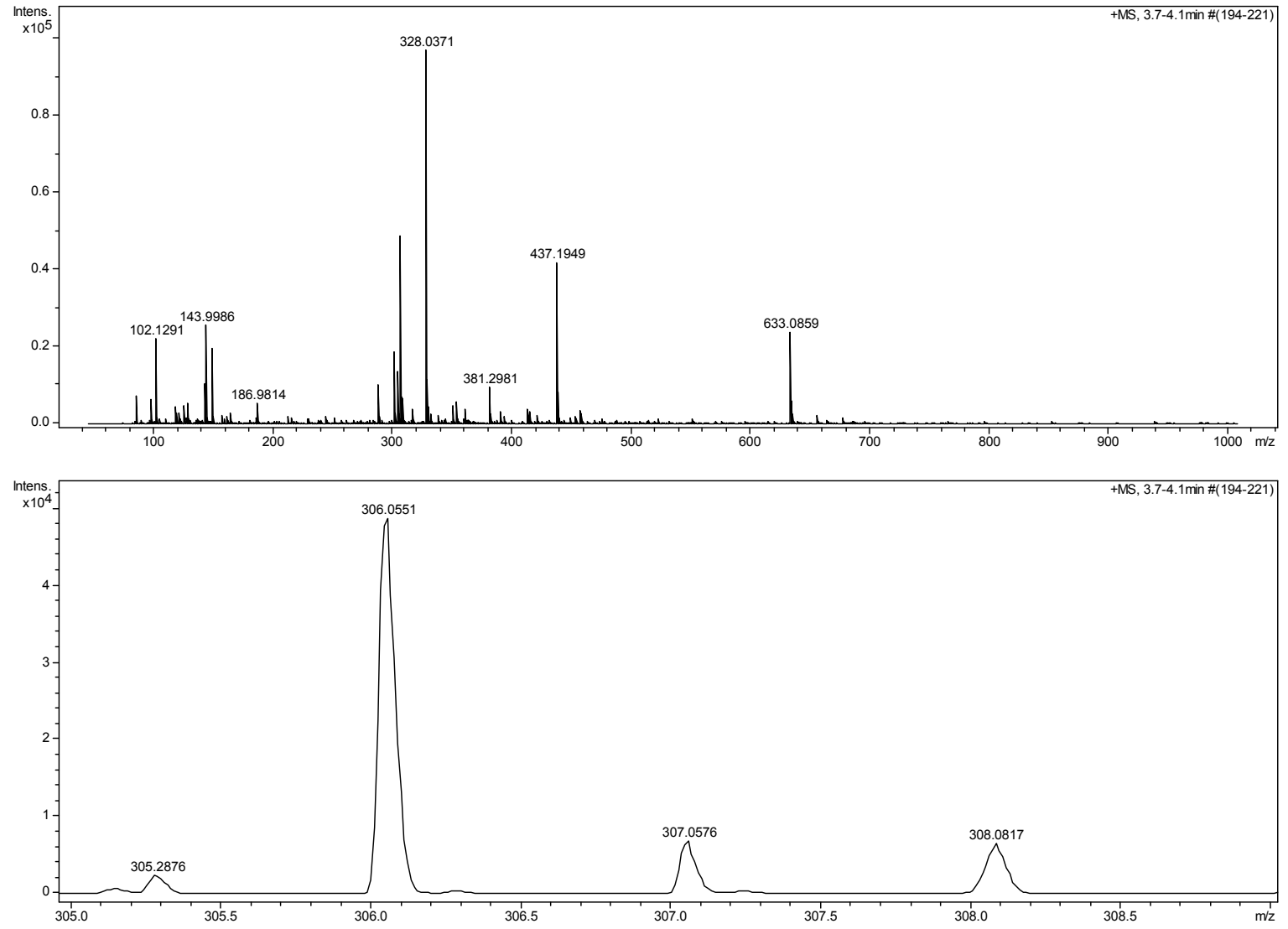
N'-(4-hydroxy-3-methoxybenzylidene) benzenesulfonyl hydrazone (13)
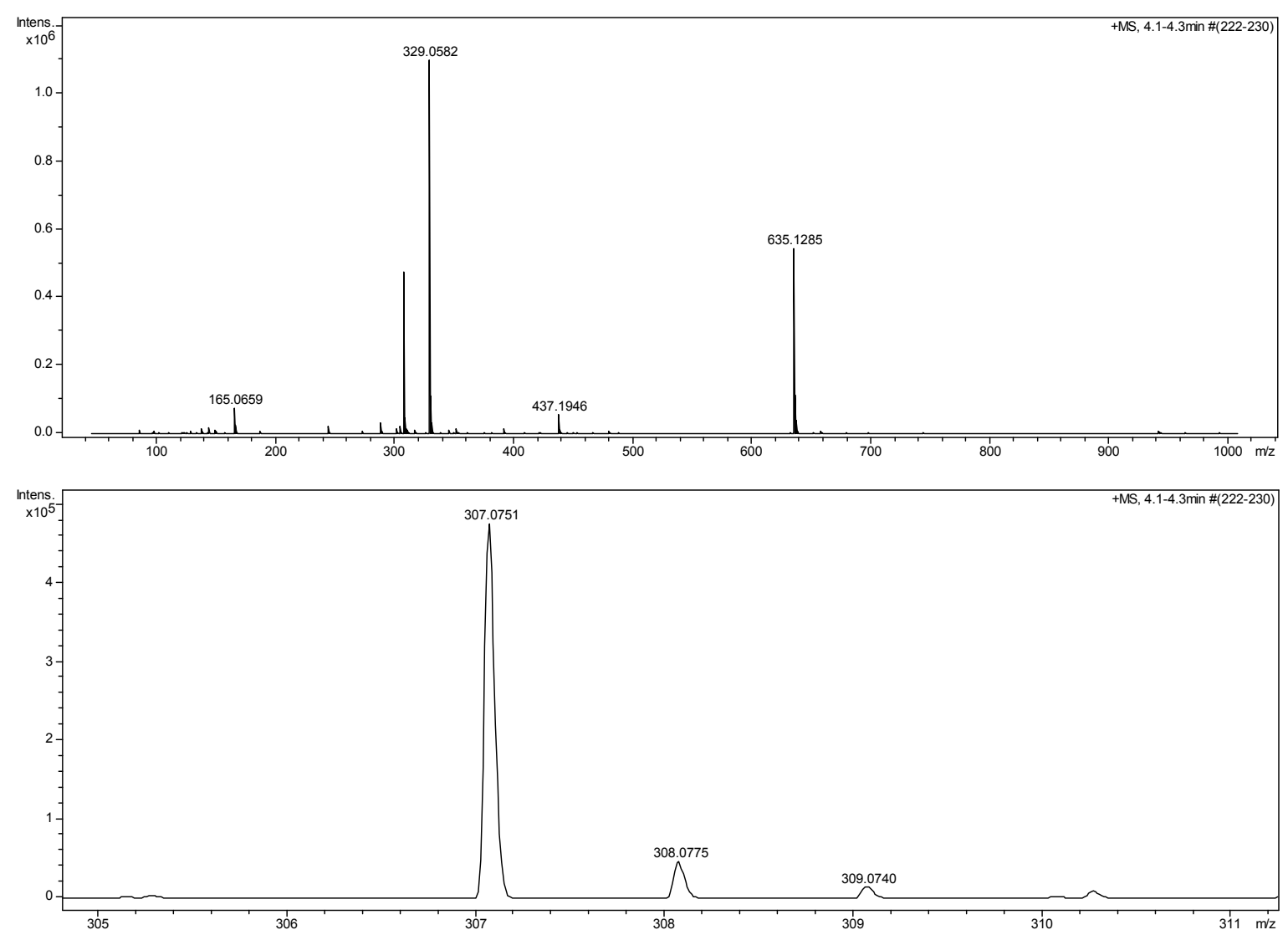


\section{References}

1 K. Shyam, L. A. Cosby and A. C. Sartorelli, J. Med. Chem., 1985, 28, 149-152.

2 M. T. Tavares, K. F. M. Pasqualoto, J. Van-de-Streek, A. K. Ferreira, R. a. Azevedo, M. C. F. C. B. Damião, C. P. Rodrigues, P. L. De-Sá-Júnior, J. a. M. Barbuto, R. Parise-Filho and F. F. Ferreira, J. Mol. Struct., 2015, 1088, 138-146.

3 J. A. P. Sato, F. N. Costa, M. D. da Rocha, E. J. Barreiro, C. A. M. Fraga, F. Punzo and F. F. Ferreira, CrystEngComm, 2014, 17, 165-173.

4 T. F. Silva, W. Bispo Júnior, M. S. Alexandre-Moreira, F. N. Costa, C. Monteiro, F. Furlan Ferreira, R. C. R. Barroso, F. Noël, R. T. Sudo, G. Zapata-Sudo, L. M. Lima and E. Barreiro, Molecules, 2015, 20, 3067-3088.

5 A. A. Coelho, J. Evans, I. Evans, A. Kern and S. Parsons, Powder Diffr., 2011, 26, S22 S25.

6 W. I. F. David, K. Shankland, J. van de Streek, E. Pidcock, W. D. S. Motherwell and J. C. Cole, J. Appl. Crystallogr., 2006, 39, 910-915.

7 H. M. Rietveld, Acta Crystallogr., 1967, 22, 151-152.

8 H. M. Rietveld, J. Appl. Crystallogr., 1969, 2, 65-71. 